\title{
APLICACIÓN DE LAS MEDIDAS CLÁSICAS DE PERFORMANCE EN LOS FONDOS DE INVERSIÓN BRASILEÑOS DE RENTA VARIABLES*
}

\author{
ALEXANDRO BARBOSA \\ Doctorando en Contabilidad y Finanzas \\ Universidad dẻ Zaragoza - España \\ Bolsista do Governo Brasileiro CAPES \\ E-mail:546060@unizar.es
}

\author{
JOSÉ LUÍS SARTO MARZAL \\ Professor del Doctorado en Contabilidad y Finanzas \\ Universidad de Zaragoza - España \\ E-mail: jlsarto@unizar.es
}

\section{RESUMEN}

En este estudio realizamos una aplicación de las medidas clásicas de performance de carteras y sus respectivas alternativas de coherencia absoluta con los datos de las rentabilidades mensuales de los fondos de inversión brasileños de renta variable en el período comprendido entre julio/2003 a julio/2005. Con el objetivo de desarrollar este estudio utilizamos el análisis multivariante para clàsificar los fondos conforme sus caracteristicas de volatilidad así como identificar el conjunto de fondos con mayores variaciones. Para el análisis econométrico-financiero utilizamos las aportaciones de Sharpe, Treynor y Jensen y sus respectivas medidas de coherencia absoluta adaptadas por Ferruz y Sarto. Por último, realizamos un estudio empírico, demostrando que, en términos estadísticos, los rangos de clasificaciones de los distintos índices clásicos de performance son notoriamente similares, mientras que los rangos establecidos por las alternativas de coherencia absoluta para las tres medidas clásicas de performance generaron similitudes menos importantes.

Palabras clave: Fondos de Inversión de Renta Variable; Rentabilidad-Riesgo; Teorías Clásicas de Performance; Análisis multivariante; Análisis econométrico-financiero.

\section{ABSTRACT}

In this study we made an application of the classical measure of performance of portfolio and their respective alternatives of absolute coherence with the monthly dates of the profitability of the brasilian variable income fund during the period of July/2003 and July/2005. In order to develop this study we use the multivariate analysis to classify the funds according to their characteristics of volatility and identify the group of funds with the higher variation. When we made the econometric-finance analysis we used the contribution of Sharpe, Treynor and Jensen and their respective measures of absolute coherence adapted by Sarto and Ferruz. Finally, we made an empirical study that demonstrate that, in statistics terms, the categories of classification of the different classical performance are notoriously similar whereas the categories established by the alternatives of absolute coherence for the three classical measure of performance generated similarities less important.

Keywords: Variable Income Fund of Investment; Profitability-Risk; Theory of Classical Performance; Multivariate Analysis; Econometric-Finance Analysis. 


\section{INTRODUCCIÓN}

Actualmente el nivel de desarrollo del mercado de capitales es un importante indicador del nivel de desarrollo económico de un determinado país, una vez que los inversores de los diferentes países buscan invertir en mercados fuertes y que generen un mayor rendimiento junto a un nivel menor de riesgo. En este sentido, los participantes evalúan las rentabilidades de las empresas diariamente de acuerdo con la información emitida por el mercado y por las propias empresas.

Bajo la óptica del inversor del mercado de capitales que aplica recursos en diversos tipos de activos financieros, lo que cuenta no es la rentabilidad individual de cada activo, sino la contribución de cada activo financiero en la minimización del riesgo total asociada a la rentabilidad total de la cartera.

Tal preocupación surgió a partir de las teorías clásicas de performance de carteras iniciadas por el modelo propuesto por Markowitz (1952) que después fue simplificado por Sharpe (1963). Un año después, Sharpe (1964) desarrolló el modelo denominado de Capital Asset Pricing Model (CAPM).

Estas ideas iniciales servirán de base para otros estudios clásicos que aportaron índices de performance de las carteras. Principalmente, destacan el ratio premio-volatilidad de Treynor(1965), el ratio premio-variabilidad de Sharpe (1966) y la rentabilidad diferencial de Jensen (1968), que serán comentados en el siguiente apartado.

En Brasil en $1957^{1}$ los participantes del mercado empezaron a constituir condominios con capitales de distintos inversores, para aplicar sus recursos, poniendo en marcha el funcionamiento del primer fondo de inversión.

Actualmente el mercado bursátil brasileño é considerado lo mayor centro de negocios de accciones de Latino Améri$\mathrm{Ca}^{2}$ que atrae importantes niveles de inversiones y sus respectivos riesgos de mercado, lo que proporciona un mayor interés en estudios empíricos realizados en el mercado Brasileño que trate de las medidas de performance de carteras.
Aunque las aportaciones de Sharpe, Treynor y Jensen surgieron del modelo pionero de Markowitz y sus componentes básicos de rentabilidad y riesgo, se hace necesario investigar se estas medidas ofrecen valoraciones que correspondiesen a rangos similares para la performance de los fondos de inversión.

Podemos encontrar en la literatura algunos trabajos que intentan mejorar las medidas clásicas de performance. En particular, Ferruz y Sarto (1997a, 1997b) apuntan ciertas inconsistencias de estas medidas tradicionales en determinados entornos al aportar evidencia empírica en los mercados españoles de fondos de inversión y de planes de pensiones. Dichos autores realizan pequeñas modificaciones de estos índices, intentando no alterar la naturaleza de las medidas de partida que permitan ofrecer clasificaciones coherentes de la eficiencia obtenida por las diferentes carteras analizadas.

La motivación de este trabajo se centra en investigar el mercado brasileño de los fondos de inversión de renta variable, observando si se producen las inconsistencias observadas por los autores anteriormente indicados $y$, si es así, utilizar los índices alternativos que proponen. Pretendemos valorar, de la forma más adecuada posible, el nivel de eficiencia de los fondos de inversión brasileños de renta variable brasileños que presentaron mayores volatilidades en sus rentabilidades medias mensuales en el período de julio/2003 a julio/2005.

Para esto el trabajo se desarrolla del siguiente modo: en la segunda sección se analizan las características de las medidas clásicas de performance para, en la tercera, se presentan las medidas de performance de coherencia absoluta; en la cuarta sección se indican las clasificaciones de los fondos de inversiones brasileños, en la quinta se detalla el diseño metodológico de la investigación, en la sexta se recogen los análisis de los datos y en la séptima y ultima son presentadas las conclusiones finales.

\section{LAS MEDIDAS CLÁSICAS DE PERFORMANCE}

Las aportaciones de Sharpe, Treynor y Jensen son consideradas como las medidas clásicas de performance. Sobre ellas, Ferruz y Sarto (1997a) comentan que en relación con la valoración de la performance de las carteras, Sharpe(1966), Treynor(1965) y Jensen (1968) fueran pioneros en su estudio. Este concepto, de modo literal, significa resultado o rendimiento ofrecido por las carteras. Sin embargo, en los estudios de los autores citados, se comprueba que el sentido financiero que aportan a la idea de performance es superior o más completo que el simple análisis de la rentabilidad de una cartera de activos financiero.

Las expresiones que corresponden a las medidas clásicas de performance son:

El ratio premio-volatilidad de Treynor

$$
T_{p}=\frac{E_{p}-R_{f}}{\beta_{p}}
$$

1 Según información contenida en el sitio www.anbid.com.br de la Associação Nacional dos Bancos de Investimento - ANBID

2 Según informaciones contenidas en el sitio www.bovespa.com.br la Bolsa de Valores de São Paulo - BOVESPA se configura, en la actualidad, en el mayor centro de negocio de acciones de Latino América 
El ratio premio-variabilidad de Sharpe

$$
S_{p}=\frac{E_{p}-R_{f}}{\sigma_{p}}
$$

La rentabilidad diferencial de Jensen

$$
J_{p}=\left[E_{p}-R_{f}\right]-\left[E_{M}-R_{f}\right] \times \beta_{p}
$$

Donde:

$E_{p}=$ rentabilidad media de cada cartera, en su caso de cada fondo de inversión;

$R_{f}=$ rentabilidad media de los activos considerados sin riesgo;

$\beta_{p}=$ volatilidad de cada cartera respecto al mercado, elemento habitualmente representativo del nivel de riesgo sistemático de cada cartera; $\sigma_{p}=$ desviación típica de la rentabilidad histórica de la cartera;

$E_{M}=$ rentabilidad media del mercado.

A lo largo del tiempo estas medidas han sido tratadas en diversos estudios empíricos, con el objetivo de averiguar cómo se comportan en las más distintas situaciones, en ocasiones atípicas, surgiendo entonces aportaciones que proporcionan sugerencias de cambios en los ratios originales. De entre las alternativas propuestas, destacamos los cambios al ratio premio-variabilidad de Sharpe propuestos por Ferruz y Sarto (2003) al trabajar sobre una muestra de los fondos de inversión españoles y, más específicamente, las medidas alternativas de coherencia absoluta descritas por Sarto (1995) que serán comentadas en el apartado siguiente.

\section{LAS MEDIDAS DE PERFORMANCE DE COHERENCIA ABSOLUTA}

Como se ha indicado, centramos nuestro trabajo en la búsqueda de modificaciones que se ajusten a las más distintas situaciones que se pueden producir en los mercados. En este sentido, nos ocupan posibles escenarios de inconsistencia en los que pueda darse que $\mathrm{E}_{\mathrm{p}}<\mathrm{R}_{\mathrm{f}}$, o bien que $\mathrm{E}_{\mathrm{M}}<\mathrm{R}_{\mathrm{f}}$ o incluso que $\beta_{\mathrm{p}}<0$. Ferruz y Sarto (1997a) proponen, a partir de los estudios realizados por Sarto (1995), realizar una revisión critica de las medidas clásicas de performance de certeras y con esto proponer índices alternativos ajustados a las dichas situaciones, permitiendo así la valoración de la gestión de las carteras formadas por activos financieros en cualquier escenario, tanto si no curre ninguna de las situaciones anómalas planteadas, como si sucede una de ellas, como si concurren varias simultáneamente.

Las expresiones planteados por los dichos autores son:

Alternativa de coherencia absoluta al índice de Sharpe

$$
S_{p}^{*}=\frac{E_{p} / R_{f}}{\sigma_{p}}
$$

Alternativa de coherencia absoluta al índice de Treynor

$$
T_{p}^{*}=\frac{E_{p} / R_{f}}{\beta_{p}}
$$

Alternativa de coherencia absoluta al índice de Jensen

$$
J_{p}^{*}=\frac{E_{p}}{R_{f}}-\frac{E_{M}}{\beta_{p}} \times \beta_{p}
$$

La aplicación empírica de las dichas alternativas de coherencia, nos proporcionará otra elección de evaluación de la performance de los fondos de inversión de renta variable brasileños, y con esto ayudar en la evaluación final del presente estudio, presentando, así, una respuesta alternativa al problema impuesto por la investigación.

\section{CLASIFICACIONES DE LOS FONDOS DE INVERSIONES BRASILEÑOS}

Actualmente hay en Brasil dos organismos que establecen clasificaciones de los fondos de inversiones brasileños que son: la "Comissão de Valores Mobiliários - CVM", por medio de la instrucción CVM numero 409 (clasificación reglamentaria) y la "Associação Nacional dos Bancos de Investimento - ANBID", siendo la primera clasificación más genérica, mientras que la segunda es mucho más detallada y restrictiva.
Según la Bolsa de Valores de São Paulo (2006), el proceso de clasificación de los fondos de inversión establecida por la CVM, se completa con la clasificación ANBID, siendo ésta más restrictiva. En este sentido, es posible garantizar una correcta compatibilidad entre las base de datos ANBID y CVM.

La Bolsa de Valores de São Paulo (2006), aún destaca que la compatibilidad de las dichas clasificaciones se hace

3 Corresponde a la comisión de valores mobiliarios brasileña.

4 Corresponde a la Asociación brasileña de Bancos de Inversión y es considerada como la principal representante de las empresas que actúan en el mercado bursátil brasileño. 
necesaria para se evitar que divulgaciones de informaciones conflictivas, entre clases CVM y categorías ANBID, confundan los inversores y proporcionen pérdidas en la industria de los fondos en general.

Las afirmaciones nos llevan a creer que no hay incompatibilidad entre las dos clasificaciones. Los datos utilizados en la investigación fueron sacados del sistema SMART FUND $^{\circ 5}$, que utiliza las clasificaciones propuestas por la ANBID. Por ello, llevaremos a cabo la investigación conforme a dicha clasificación según la Tabla $1 \mathbf{0}$.

En la tabla se recogen todas las clasificaciones ANBID, y resumidamente sus conceptos en términos de riesgo. Tal clasificación es de fundamental importancia para identificar las distintas clases de riesgo de los fondos de inversión en función de sus rentabilidades mensuales conocidas.

Aún cabe destacar que los fondos de inversión de categoría "Corto Plazo", "Referenciados" y "Renta Fija", son de renta fija. En este sentido no serán contemplados por el actual estudio, pues como hemos dicho, pretendemos realizar solamente una evaluación de los fondos de inversión de renta variable, es decir: "Multimercados", "Inversión en el exterior", "IBOVESPA", "IBX”, "Acciones Sectoriales", "Acciones otros" y "Cambio".

Tabla 1 \ Clasificación de los fondos según la ANBID

\begin{tabular}{|c|c|c|}
\hline Categoría & Tipo ANBID & Riesgos \\
\hline Corto Plazo & Corto Plazo & DI/SELIC \\
\hline \multirow{2}{*}{ Referenciados } & Referenciado DI & \multirow{2}{*}{ Índex de Referencia } \\
\hline & Referenciado Otros & \\
\hline \multirow{4}{*}{ Renta Fija } & Renta Fija & Interés \\
\hline & Renta Fija Crédito & Interés + Crédito \\
\hline & Renta fija Múltiple-índices & Interés + Crédito + Índice de precios \\
\hline & Renta fija con Apalancamiento & Interés + Crédito + Índice de precios + Apalancamiento \\
\hline \multirow{6}{*}{ Multimercados } & Balanceados & \multirow{6}{*}{ Diversas Clases de activos } \\
\hline & Multimercados sin RV & \\
\hline & Multimercados con RV & \\
\hline & Multimercados sin RV con Apalancamiento & \\
\hline & Multimercados con RV con Apalancamiento & \\
\hline & Capital Protegido & \\
\hline Inversión en el exterior & Inversión en el exterior & Título de la deuda externa y tasa de cambio \\
\hline \multirow{3}{*}{ IBOVESPA } & Acciones IBOVESPA Indexado & \multirow{2}{*}{ Índice de Referencia. } \\
\hline & Acciones IBOVESPA Activos & \\
\hline & Acciones IBOVESPA Activo Apalancado & Índice de Referencia + Apalancamiento \\
\hline \multirow{3}{*}{$\mathrm{IBX}$} & Acciones IBX Indexado & \multirow{2}{*}{ Índice de Referencia } \\
\hline & Acciones IBX Activos & \\
\hline & Acciones IBX Activo con Apalancamiento & Índice de Referencia + Apalancamiento \\
\hline \multirow{2}{*}{ Acciones Sectoriales } & Acciones Sectoriales Telecomunicaciones & \multirow{2}{*}{ Riesgo del Sector } \\
\hline & Acciones Sectoriales Energía & \\
\hline \multirow{2}{*}{ Acciones otros } & Acciones otros & - \\
\hline & Acciones otros con Apalancamiento & Apalancamiento \\
\hline \multirow{6}{*}{ Cambio } & Cambio Dólar Indexado & \multirow{3}{*}{ Moneda de Referencia } \\
\hline & Cambio Euro Indexado & \\
\hline & Cambio Dólar sin Apalancamiento & \\
\hline & Cambio Dólar con Apalancamiento & Moneda de Referencia + Apalancamiento \\
\hline & Cambio Otros sin Apalancamiento & Variación de las Monedas \\
\hline & Cambio Otros con Apalancamiento & Variación de las Monedas + Apalancamiento \\
\hline
\end{tabular}

Fuente: Associação Nacional dos Bancos de Investimento (2006, traducción nuestra)

\section{DISEÑO DE LA INVESTIGACIÓN}

\subsection{Clasificación de la investigación}

Esta investigación es de tipo descriptivo, de análisis de los hechos, así como de su interpretación, registro y clasificación. De ese modo, partimos de una amplia investigación bibliográfica de la teoría clásica y actual sobre performance de carteras ya presentada previamente y propia del carácter científico de este trabajo.

La investigación bibliográfica fue realizada con el objetivo de establecer un planteamiento teórico sobre la problemática del estudio para dar consistencia a las constata- 
ciones demostradas a lo largo de ese trabajo. Así, en esto proceso, fueran consultados libros técnicos especializados, tesis y artículos técnicos en revistas especializadas e investigación en la Internet, todo esto para la evaluación de la aplicación de las medidas clásicas de performance de cartera y sus respectivas alternativas de coherencia absoluta en el conjunto de fondos de inversión de renta variable brasileños que presentaran mayores variaciones en las rentabilidades mensuales medias en el período de julio/2003 a julio/2005, caracterizando así una investigación de verificación analítica y empírica.

Así, en el proceso del trabajo, fue aplicada la técnica estadística de análisis multivariante para la selección y análisis de los datos, lo que derivó en una investigación de naturaleza cuantitativa. Para ello se utilizó el programa SPSS $^{\odot}$ for windows 6 , versión 12.0 así como Excel $^{\circledR} 2003^{7}$.

\subsection{Construcción de las bases de datos}

La base de datos utilizada en la investigación se compone de las siguientes fuentes:

$1^{\text {a) }}$ Las rentabilidades mensuales de los fondos de inversión de renta variable fueran obtenidas del sistema SMART FUND ${ }^{\circ}$, en la cual fueron extraídos todos los datos de todos los fondos de inversión de renta variable en el período que comprende de julio/2003 a julio/2005, es decir, un total de 25 períodos. Inicialmente, fueron considerados todos los fondos de inversión de renta variable brasileños, sin embargo un análisis de los cortes transversales nos hizo eliminar los fondos que no disponían de datos de rentabilidad mensual en alguno de los veinticinco períodos de la serie temporal, restando así un total de 1.184 fondos de inversión de renta variable seleccionados.

$2^{\mathrm{a}}$ ) La información de la rentabilidad periódica del activo financiero libre de riesgo fue sacada de la Cámara de Custodia y Liquidación - CETIP. Se tomó el activo Tasa DI-CETIP Over (Extra-Grupo), cuya rentabilidad es calculada y divulgada por la CETIP a partir de las operaciones de emisiones de Depósitos Ínter financieros fijados previamente, pactadas por un día útil y registradas y liquidadas por el sistema CETIP, de acuerdo con la determinación del Branco Central Brasileño (CÂMARA DE CUSTÓDIA E LIQUIDAÇÃO, 2006).

$3^{\text {a) }}$ Los datos de rentabilidad del mercado de valores brasileño, para lo cual se han tomado como referencia los índices generales de la BOVESPA, sacados de su sistema de divulgación de datos en su página web ${ }^{8}$.

\subsection{Clasificación de los conjuntos de fondos}

La clasificación de los grupos se hace importante a partir del momento que se necesitan identificar conjuntos homogéneos de datos. El objetivo es separar los fondos de inversión de renta variable en submuestras de carteras similares a partir de las 25 rentabilidades mensuales estudiadas.

Se pretende identificar el grupo de fondos de inversión con mayores niveles medios de rentabilidades mensuales, para esto se realizó un análisis multivariante de datos denominada análisis clusters, que fue dividido en cuatro etapas.

$\left.1^{\mathrm{a}}\right)$ Inicialmente se realizó un análisis para identificar la cantidad adecuada de grupos por medio de los análisis de conglomerados jerárquicos del SPSS, utilizándose el criterio de vinculación inter-grupos y los niveles de correlación de Pearson. Se utilizó, también, la opción de transformación de medidas para valores absolutos. Según Salvador y Gargallo (2006) "Conviene, por tanto, utilizar un método jerárquico para obtener una clasificación inicial de los datos que luego puede ser refinada por otros métodos."

$2^{\text {a) }}$ Tras la primera etapa se trasladaron los datos obtenidos a la hoja de calculo Excel, para la identificación gráfica del numero adecuado de grupos, buscando grandes saltos en el historial de conglomeración (SALVADOR y GARGALLO, 2006).

$\left.3^{a}\right)$ Después de la definición de la cantidad de grupos, se procede a la clasificación de los grupos en dos fases: utilizado como variables continuas las rentabilidades mensuales, utilizándose de la log-verosimilitud como medida de distancia para el numero fijo de grupos establecidos en la etapa anterior, y finalmente se crea la variable del grupo de pertenencia. Adicionalmente se realiza el análisis de la significatividad de cada variable en la formación del clúster por medio observación gráfica del ajuste de Bonferroni aplicado que utiliza el estadístico $T$ de Student.

$4^{\mathrm{a}}$ ) Finalmente, se realiza la identificación gráfica del grupo de mayor variación de rentabilidad media mensual, tras utilizar las herramientas de tablas de contingencia y cubos OLAP del SPSS y su correspondiente traslado a la hoja de cálculo Excel.

\subsection{Análisis econométrico-financiero}

Siguiendo con el proceso de investigación, el siguiente paso es la realización del análisis econométrico-financiero de los fondos de inversión que pertenecen al grupo con mayores variaciones de las rentabilidades medias mensuales y, posteriormente, separar y analizar la información de dichos fondos con el objetivo de identificar eventuales inconsistencias en los datos.

Para los cálculos de los betas $\left(\beta_{\mathrm{p}}\right)$ de los fondos de inversión se efectuaron regresiones individuales de cada fon-

\footnotetext{
6 SPSS - Statistical Package for the Social Sciences, es un programa utilizado por las ciencias sociales para tabulación, análisis y tratamiento de datos. Copyright (C) SPSS, Inc. 7 Hoja de Cálculo. Copyright (C) Microsoft Corporation

8 www.bovespa.com.br
} 
do, de acuerdo con la información de la rentabilidad de los activos libres de riesgo $\left(R_{F}\right)$, y del índice de referencia del mercado $\left(R_{M}\right)$.

La expresión econométrica para el cálculo de los betas fue $\left(\beta_{\mathrm{p}}\right)$ :

$$
R_{p t}=\alpha_{p}+\beta_{p} x R_{M t}+\varepsilon_{p t}
$$

Donde:

$R_{p t}=$ la rentabilidad ofrecida por el título $p$ en el período t;

$\alpha_{p}=$ término independiente del modelo, expresa la parte del rendimiento del titulo $p$ que es independiente del mercado;

$R_{\mathrm{Mt}}=$ el rendimiento ofrecido por el mercado en el periodo t;

$\varepsilon_{\mathrm{pt}}=$ la perturbación aleatoria del modelo econométrico-financiero planteado. Se refiere a la parte de la rentabilidad restante que no se explica por el modelo debido, por tanto, a otros factores no contemplados por el modelo.

Tras construir la base de datos de las variables utilizadas en el análisis econométrico-financiero se procede a los cálculos del ratio premio-volatilidad de Treynor, el ratio premio-variabilidad de Sharpe, la rentabilidad diferencial de Jensen, según las expresiones contenidas en el apartado 2. Así como, los cálculos de la medidas alternativas de coherencia absoluta al índice de Sharpe, de Treynor y de Jensen, según las expresiones contenidas en el apartado 3.

Finalmente, para poder establecer el grado de correlación y, consecuentemente, el nivel de similitud entre los distintos rangos, se procede a la aplicación del coeficiente de correlación de rango de Spearmen⿳⺈, que fue calculado por medio de la herramienta de análisis de correlación bivariada del SPSS.

\subsection{Hipótesis metodológicas}

Las hipótesis metodológicas corresponden a respuestas anticipadas al problema propuestas por la investigación. Nos basamos de estudios anteriores, en particular de Ferruz y Sarto (1997a) "Si el objetivo inicial de las medidas de performance de carteras es establecer un valor de la misma para cada una de las carteras analizadas, el objetivo último de estos índices es establecer un ranking de clasificación de las carteras, de modo que permita analizar de forma comparada si una determinada cartera ha estado mejor o peor gestionada que otra. En esto sentido, se trata de realizar clasificaciones o rankings de gestión de un conjunto de carteras".

Así, tras verificar el problema de investigación y las aportaciones anteriores, se parte de las siguientes hipótesis metodológicas:

Hipótesis Metodológica 1: El grupo constituido por los fondos de inversión de renta variable que presenta mayor variación de las rentabilidades medias mensuales en el período comprendido entre julio/2003 a julio/2005, tras los cálculos de las tres medidas clásicas de performance de carteras, obtiene rangos de gestión estadísticamente similares entre las tres medidas.

De acuerdo con las aportaciones comentadas en el apartado 3, las alternativas de coherencia absoluta permiten la valoración de la gestión de las carteras formadas por activos financieros en cualquier escenario, tanto si existe alguna situación anómala como si no.

Hipótesis Metodológica 2: El grupo constituido por los fondos de inversión de renta variable que presenta mayor variación de las rentabilidades medias mensuales en el período comprendido entre julio/2003 a julio/2005, tras los cálculos de las alternativas de coherencia absoluta para las tres medidas clásicas de performance de carteras, obtiene rangos de gestión estadísticamente similares entre las tres medidas.

Con esto creemos que la investigación se ajusta a cualquier escenario, incluso el brasileño lo que permitió una evaluación bajo dos perspectivas.

\section{APLICACIÓN EMPÍRICA}

Tras la explicación de todo el diseño de la investigación, se procede a la aplicación empírica propiamente dicha con los resultados de los análisis estadísticos de clasificación de los conglomerados, los análisis econométrico-financieros y por último, los contrates de las hipótesis metodológicas con la aplicación de los índices clásicos y de las alternativas de coherencia absoluta, todo esto para buscar el cumplimiento del objetivo final de la investigación.

\subsection{Clasificación de los conglomerados}

En el apartado 5.3 se ha detallado todo el tratamiento estadístico de la clasificación de los conglomerados, en el caso de la presente investigación, se llega a una distribución adecuada en tres conglomerados, correspondiendo a tres grupos con comportamientos diferentes (altas, medias y bajas), en relación al nivel de variaciones de las rentabilidades medias mensuales. Aún destacamos que las variables (cada una de las 25 rentabilidades mensuales del período comprendido entre julio/2003 a julio/2005), tras la observación gráfica de las ubicaciones del estadístico T de Student en el ajuste de Bonferroni aplicado para cada conglomerado, se constata que todas son significativas una vez que todos los valores T excedieron la línea de guiones positiva.

9 Es una técnica estadística no paramétrica para evaluar el grado de relación entre dos variables, cuando los datos se disponen en rangos. Su objetivo es el cálculo de un coeficiente que determina el nivel en el que dos conjuntos de rangos concuerdan. (STEVENSON, 2000) 
La Tabla 20 establece la ubicación de las categorías de fondos de inversión en sus respectivos conglomerados (grupos).

Como se observa, el grupo 1 está compuesto, básicamente, por las categorías Acciones otros, Acciones Sectoriales, IBOVESPA e IBX. El grupo 2 compuesto por los fondos de inversión de las categorías de Cambio e Inversión en el exterior, y por ultimo los del grupo 3 que corresponden a los de categoría multimercados.

Los agrupamientos presentados en la tabla también nos presentan una idea de cómo es la adherencia de los conglomerados calculados conforme a las rentabilidades medias mensuales y a los tipos de riesgos de cada categoría. En este sentido, pertenecen al grupo 1 las categorías que poseen fondos de inversión con componentes de riesgos mas variados, principalmente los que toman como referencia los índices BOVESPA e IBX con y sin apalancamiento. Los que pertenecen al grupo 2 tienen como riesgos las tasas de cambio y sus respectivas variaciones de la moneda de referencia y por fin los del grupo 3 buscan el retorno a través de inversiones en diversas clases de activos, diversificando su riesgo.

Así cada grupo queda distribuido con las siguientes cantidades: el grupo 1 con 286 fondos de inversión, el grupo 2 con 125 fondos de inversión y el grupo 3 con 773 fondos de inversión, totalizando 1.184 fondos.

Tras esto procedemos a calcular las rentabilidades medias de cada conglomerado donde se identifica gráficamente el conglomerado que será tomado como base para la aplicación del análisis econométrico-financiero, siendo éste el grupo que presenta mayor variación en las rentabilidades medias mensuales.

En el Grafico $1 \oslash$ se presentan las líneas de comportamiento de la rentabilidad media mensual para cada conglomerado (grupo) de fondos de inversión de característica variable.
Según la visualización gráfica, se observa que el grupo 1 es el de mayor variación, y consecuentemente mayor riesgo. En este sentido, a este grupo le será hecha la evaluación de los índices clásicos de performance de cartera y sus alternativas de coherencia absoluta, pasándose a continuación a su análisis econométrico-financiero en el siguiente apartado.

\subsection{Análisis econométrico-financiero}

A continuación se procede a evaluar la eficiencia en la gestión de los fondos de inversión, de acuerdo con lo indicado en los apartados 5.4 y 5.5. En esta etapa es aplicado el modelo econométrico-financiero y son calculadas todas las variables (apéndice 01) integrantes de las expresiones que incluyen los índices clásicos de performance y las alternativas de coherencia absoluta.

La rentabilidad media del activo financiero sin riesgo $\left(R_{\mathrm{f}}\right)$ para los 25 períodos comprendidos entre julio/2003 y julio/2005, en esto caso la DI-CETIP es 1,4116\% y la rentabilidad periodal media del mercado brasileño - índice BOVESPA $\left(\mathrm{R}_{\mathrm{M}}\right)$ calculada para el mismo periodo es de 2,0364\%.

En relación al modelo econométrico que fue desarrollado para el calculo del beta $\left(\beta_{\mathrm{p}}\right)$, nos ha proporcionado resultados bastante satisfactorios (apéndice 01), pues se observa que cerca de $96 \%$ de los fondos presenta valores de $\mathrm{R}^{2}$ Ajustado con potencia explicativa superior al $50 \%$, mientras que el $79 \%$ de los $\mathrm{R}^{2}$ Ajustados de las regresiones supera el $75 \%$ de explicación del modelo.

Observando el estadístico T de Student, todos los fondos rechazan la hipótesis nula con un nivel se significación de $95 \%$, o sea el modelo estadístico funciona aceptablemente en su potencia explicativa, y consecuentemente garantiza una fuerte validez para los cálculos de los betas.

A continuación serán calculados los índices de Sharpe, Treynor y Jensen.

Tabla 2 || Distribución de los tipos de fondos en los conglomerados

\begin{tabular}{l|l|c}
\multicolumn{1}{c|}{ Categoría } & \multicolumn{1}{|c}{ Riesgos } & $\begin{array}{c}\text { Conglomerado } \\
\text { (Grupo) }^{\mathbf{1 0}}\end{array}$ \\
\hline Acciones otros & Apalancamiento & 1 \\
\hline Acciones Sectoriales & Riesgo del Sector & 1 \\
\hline IBOVESPA & $\begin{array}{l}\text { Índice de Referencia } \\
\text { Índice de Referencia + Apalancamiento }\end{array}$ & 1 \\
\hline IBX & $\begin{array}{l}\text { Índice de Referencia } \\
\text { Índice de Referencia + Apalancamiento }\end{array}$ & 1 \\
\hline \multirow{3}{*}{ Cambio } & $\begin{array}{l}\text { Moneda de Referencia } \\
\text { Moneda de Referencia + Apalancamiento } \\
\text { Variación de las Monedas } \\
\text { Variación de las Monedas + Apalancamiento }\end{array}$ & 2 \\
\hline Inversión en el exterior & Título de la deuda externa y tasa de cambio & 2 \\
\hline Multimercados & Diversas Clases de activos & 3
\end{tabular}

10 Eventualmente algunos fondos de inversión no pertenecen al mismo conglomerado establecido para el tipo de fondo. En este caso, cada fondo fue integrado en el grupo en el que situó en la mayoría de los casos. 


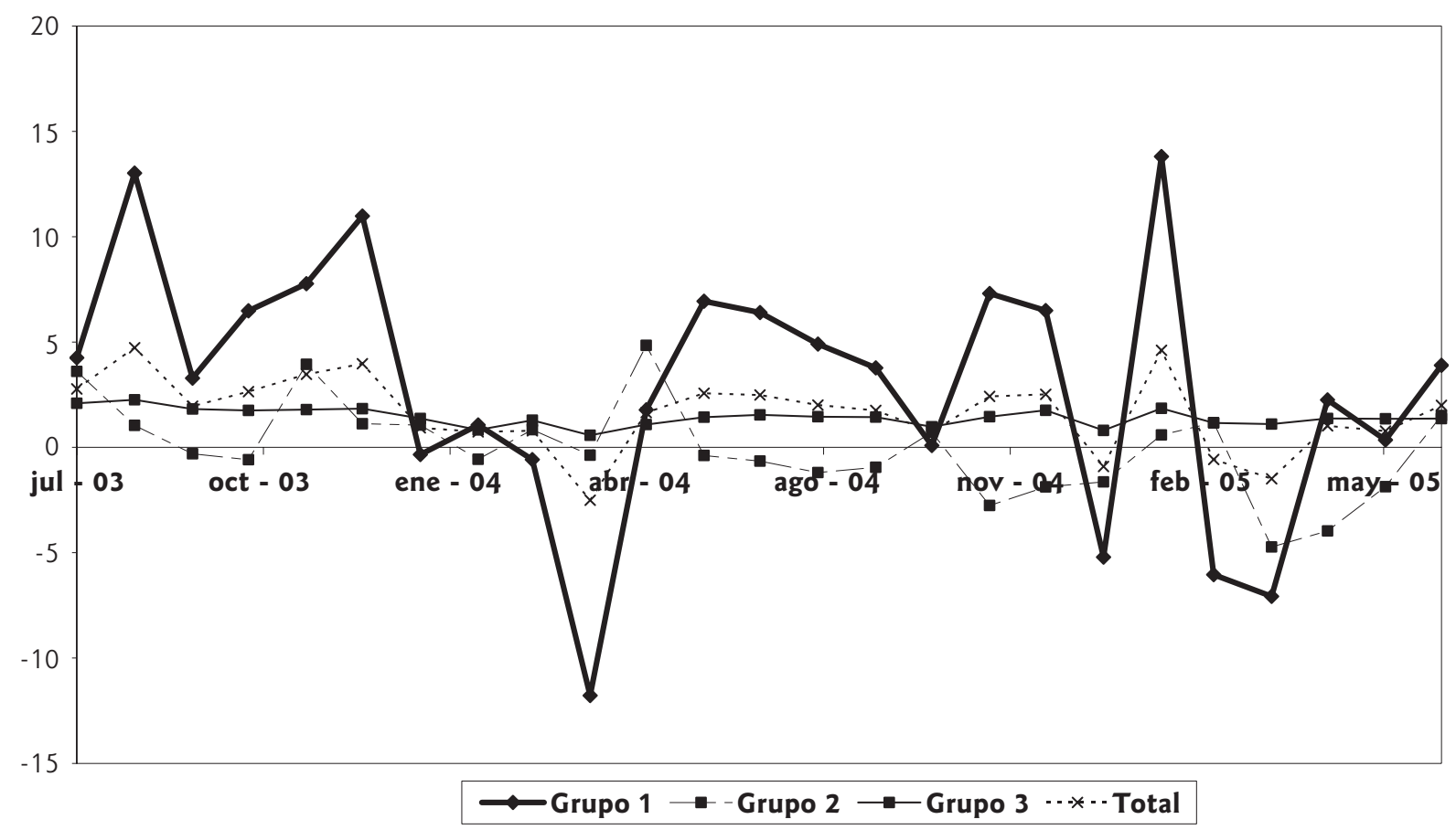

Grafico 1 - Comportamiento de la rentabilidad media mensual para cada conglomerado (grupo)

\subsection{Aplicación de los índices de Sharpe, Treynor y Jensen}

Los resultados de la aplicación de los índices de Sharpe, Treynor y Jensen se incluyen en el apéndice 01. En el mismo apéndice están presentados también los rangos de clasificación para los dichos índices.

En un proceso de evaluación individual de cada índice clásico, solo hemos encontrado importantes inconsistencias en el establecimiento de los rangos clasificatorios en dos fondos (MULTI STOCK FUNDO DE INVEST EM ACOES y BOSTON TELECOM FI EM ACOES) que presentan primas de rentabilidad negativas, es decir, $\mathrm{E}_{\mathrm{p}}<\mathrm{R}_{\mathrm{p}}$, lo que permite una adecuada aplicación de los índices clásicos en el 99,3\% de la base de datos. Sin embargo, en un análisis conjunto de los rangos establecidos por los tres índices se observa que varían según el indicador, es decir que se observa que la gestión de los fondos de inversión no es evaluada de la misma manera por todos los ratios.

Los porcentuales de los fondos en que los rangos son concordantes en pareja y concordancia general, calculados a partir de los índices Sharpe, Treynor y Jensen son presentados en la siguiente tabla $\boldsymbol{\nabla}$.
Como se observa solamente $1,4 \%$ de los fondos de inversión tienen concordancias puntuales para los tres índices y lo que posee mas concordancias puntuales son los TP x JP que alcanzan un porcentual de apenas $11,5 \%$.

Entre los varios casos que hemos observado tomamos como ejemplo el fondo "BRADESCO FIA BD - BRADESCO" que en el rango Sharpe se ubica en la $31^{a}$ posición, en el índice de Treynor en la $101^{\mathrm{a}}$ y en Jensen se ubica en la $67^{\mathrm{a}}$, o sea en general las clasificaciones puntuales son muy dispares (diferencias de rangos en 282 fondos de los 286 evaluados), cuando se comparan unas con otras. Para esto se hace necesario un análisis estocástico para comprobar hasta qué punto existe relación entre los distintos rangos con la utilización del coeficiente de correlación de rango de Spearman, según está definido en el apartado 5.4.

En la Tabla $4 \bullet$ se incluyen los resultados de los coeficientes de correlación de rango de Spearman dos a dos para los rangos establecidos por los ratios de Sharpe, Treynor y Jensen.

Como se observa, al aplicar el coeficiente de correlación de rango de Spearmen, se obtienen fuertes correlaciones que varían desde 0,845 (SPxTP) hasta 0,989 (TPxJP). Esto

Tabla 3 - Concordancias puntuales de los rangos Sharpe, Treynor y Jensen

\begin{tabular}{c|c|c|c} 
& SP & TP & JP \\
SP & - & $2,4 \%$ & $3,1 \%$ \\
\hline TP & $2,4 \%$ & - & $11,5 \%$ \\
\hline SP y TP & - & - & $1,4 \%$
\end{tabular}


Tabla 4 Resultados de las correlaciones de los rangos Sharpe, Treynor y Jensen

\begin{tabular}{c|l|c|c|c} 
& & SP & TP & JP \\
SP & Coeficiente de correlación & 1,000 & $\left.0,845^{* *}\right)$ & $0,898\left(^{* *}\right)$ \\
\hline & Sig. (bilateral) & - & 0,000 & 0,000 \\
\hline & $\mathrm{N}$ & 286 & 286 & 286 \\
\hline TP & Coeficiente de correlación & $0,845\left(^{* *}\right)$ & 1,000 & $0,989\left(^{* *}\right)$ \\
\hline & Sig. (bilateral) & 0,000 & - & 0,000 \\
\hline & $\mathrm{N}$ & 286 & 286 & 286 \\
\hline JP & Coeficiente de correlación & $0,898\left(^{* *}\right)$ & $0,989\left(^{* *}\right)$ & 1,000 \\
\hline & Sig. (bilateral) & 0,000 & 0,000 & - \\
\hline & $\mathrm{N}$ & 286 & 286 & 286
\end{tabular}

** La correlación es significativa al nivel 0,01 (bilateral).

demuestra que, estadísticamente, los fondos de inversión de renta variables brasileños son valorados de forma similar por los tres índices clásicos de performance, garantizando así una homogeneidad en las medidas clásicas de performance de carteras.

\subsection{Aplicación de las alternativas de coherencia absoluta para los} indices de Sharpe, Treynor y Jensen

Tal y como se ha procedido con los resultados de la aplicación de los índices tradicionales, los resultados que corresponden a sus alternativas de coherencia absoluta y sus respectivos rangos, también son presentados en el apéndice 01.

A diferencia del proceso de evaluación individual de cada índice clásico, las alternativas de coherencia absoluta para los índices de Sharpe, Treynor y Jensen se ajustan a las faltas de coherencia (apartado 3), que solo presentan dos fondos de inversión.

Los resultados iniciales nos llevan a creer que el bajo nivel de falta de coherencia nos podría garantizar una aproximación con los resultados presentados en el apartado anterior. Sin embargo, en el análisis conjunto de los rangos establecidos por las tres alternativas de coherencia absoluta se observa que estos rangos son todavía más dispares que los presentados por la aplicación de los índices clásicos.

Los porcentuales de los fondos, en que los rangos son concordantes en pareja y concordancia general, calculados a partir de las alternativas de coherencia absoluta a los índices de Sharpe, Treynor y Jensen son presentados en la Tabla $5 \mathbf{0}$

Como se observa sólo el 0,7\% de los fondos de inversión tienen concordancias puntuales de entre los tres índices y los que poseen mas concordancias puntuales son los TPxJP que alcanzan un porcentual de apenas $11,5 \%$, sin embargo esta ultima alcanza porcentual de concordancias puntuales igual que en los rangos establecidos a partir de los índices clásicos, mientras que las demás presentan porcentuales menores.

Entre los varios casos que hemos observados tomamos como ejemplo el fondo "CLASSE A FI ACOES PREV" que en el rango alternativo al índice Sharpe se ubica en la $3^{\mathrm{a}}$ posición, en la alternativa el índice de Treynor en la $173^{\mathrm{a}}$ y en la alternativa al índice de Jensen se ubica en la $148^{\mathrm{a}}$, o sea en general las clasificaciones puntuales son más dispares que en la aplicación de los índices clásicos.

Como se ha hecho con anterioridad, se procede ahora a un análisis estadístico para comprobar el nivel de relación entre los distintos rangos, para esto se utiliza de nuevo el coeficiente de correlación de rango de Spearmen, según está definido en el apartado 5.4.

En la Tabla 60 se incluyen los coeficientes de correlación de rango de Spearman dos a dos para los rangos establecidos por los ratios alternativos de coherencia absoluta a los índices de Sharpe, Treynor y Jensen.

Confirmando el aumento de la disparidad de los rangos presentado en la Tabla 05, la aplicación de los coeficientes de correlación de rango de Spearman nos demuestra que la relación SP*xTP* se ha debilitado significativamente, pasando lo mismo con la relación $\mathrm{SP}^{*} \times \mathrm{JP}^{*}$, sin embargo la relación $T^{*} x$ JP* se ve ligeramente fortalecida.

Esto demuestra que, estadísticamente, esta muestra de estudio de los fondos de inversión de renta variable brasileños no es adecuadamente valorada por las alternativas de coherencia absoluta. Resulta más apropiado utilizar los tres índices clásicos de performance.

Tabla 5 Concordancias puntuales de los rangos de las alternativas de coherencia absoluta a los índices de Sharpe, Treynor y Jensen

\begin{tabular}{c|c|c|c} 
& $\mathbf{S P *}$ & $\mathbf{T P *}$ & $\mathbf{J P *}$ \\
$\mathbf{S P *}$ & - & $0,7 \%$ & $0,7 \%$ \\
\hline $\mathbf{T P}^{*}$ & $0,7 \%$ & - & $11,5 \%$ \\
\hline $\mathbf{S P} \mathbf{P}^{*} \mathbf{~ T P *}$ & - & - & $0,7 \%$
\end{tabular}


Tabla 6 Resultados de las correlaciones de los rangos de las alternativas de coherencia absoluta a los índices de Sharpe, Treynor y Jensen

\begin{tabular}{c|l|c|c|c} 
& & $\mathbf{S P}$ & $\mathbf{T P}^{*}$ & JP* $^{*}$ \\
$\mathbf{S P *}$ & Coeficiente de correlación & 1,000 & $\left.0,505^{* *}\right)$ & $0,572\left(^{* *}\right)$ \\
\hline & Sig. (bilateral) & - & 0,000 & 0,000 \\
\hline & $\mathrm{N}$ & 286 & 286 & 286 \\
\hline TP* & Coeficiente de correlación & $0,505\left(^{* *}\right)$ & 1,000 & $0,991\left(^{* *}\right)$ \\
\hline & Sig. (bilateral) & 0,000 & - & 0,000 \\
\hline & $\mathrm{N}$ & 286 & 286 & 286 \\
\hline JP* & Coeficiente de correlación & $0,572\left(^{* *}\right)$ & $0,991\left(^{* *}\right)$ & 1,000 \\
\hline & Sig. (bilateral) & 0,000 & 0,000 & - \\
\hline & $\mathrm{N}$ & 286 & 286 & 286
\end{tabular}

** La correlación es significativa al nivel 0,01 (bilateral).

\section{CONCLUSIONES}

En este trabajo partimos de las aportaciones de Markowitz, Sharpe, Treynor y Jensen, en especial de sus índices clásicos de performance de carteras que vienen siendo testados empíricamente y con esto proporcionando importantes contribuciones a los avances de las teorías financieras que analizan la relación rentabilidad-riesgo y su conversión en un parámetro que posibilite la medida de la eficiencia en la gestión de las carteras.

Los fondos de inversión brasileños son clasificados por la CVM, sin embargo la ANBID intentando una mayor uniformidad y facilidad de tipificación, propone una clasificación más detallada que, al ser sintetizada, se ajusta a la clasificación definida por la CVM.

Mediante la utilización del método estadístico de análisis de clusters en las rentabilidades mensuales de los fondos de inversión de renta variable, se han podido identificar tres grupos de fondos de inversión de renta variable, en los cuales se observa una cierta adherencia entre los grupos y los tipos de riesgos a que están sometidos: un primer grupo que se presenta como un grupo de mayor variación de las rentabilidades medias, el segundo que se presenta con variaciones más moderadas de sus rentabilidades medias y por fin un tercer grupo que presenta débiles variaciones medias de sus rentabilidades mensuales.

Cuando se procede a los análisis econométricos-financieros en los fondos de inversión de renta variable brasileños que presentaron mayores variaciones de sus rentabilidades medias mensuales, se observa una elevada capacidad explicativa de las regresiones (de las que se calculan las betas). Sólo dos fondos de inversión presentan primas de rentabilidad negativa, lo que facilita la evaluación conjunta de los rangos establecidos para los fondos de inversión.

Con la aplicación de las medidas clásicas de performance planteadas por Sharpe, Treynor y Jensen, se observa que en términos estadísticos la clasificación de la gestión conforme las distintas medidas son correspondidas por fuertes similitudes con potencias explicativas de $84,5 \%, 89,8 \%$ y
98,9\% para las relaciones entre SP x TP, SP x JP y TP x JP respectivamente.

Los resultados garantizan la aceptación de la primera hipótesis metodológica en que el grupo constituido por los fondos de inversión de renta variable que presenta mayor variación de las rentabilidades medias mensuales en el período comprendido entre julio/2003 a julio/2005, tras los cálculos de las tres medidas clásicas de performance de carteras, obtiene rangos de gestión estadísticamente similares entre las tres medidas.

Al aplicar las alternativas de coherencia absoluta para las tres medidas clásicas se observan comportamientos similares a los presentados por los índices clásicos. Por su parte, se observa una potencia de similitud más débil para $\mathrm{SP}^{*} \mathrm{xTP} \mathrm{P}^{*}$ con potencia explicativa de $50,5 \%$ y SP* $\mathrm{xJ} \mathrm{P}^{*}$ con potencia explicativa de $57,2 \%$, resultados que no nos garantizan un argumento para aceptar la segunda hipótesis como aceptamos la primera. Es decir, que la performance del grupo constituido por los fondos de inversiones de renta variable que presenta mayor variación de las rentabilidades medias en el período comprendido entre julio/2003 a julio/2005, tras los cálculos de las alternativas de coherencia absoluta para las tres medidas clásicas de performance de carteras, obtiene rangos de gestión similares entre las tres medidas.

Las medidas alternativas de coherencia absoluta fueron sugeridas para su utilización en entornos con significativas inconsistencias, circunstancia que no sucede en el presente estudio.

Estudios futuros aún pueden ser realizados utilizándose del análisis discriminante como modelo predictivo de clasificación de los fondos, por lo cual futuros fondos podrían ser preclasificados por medio de tal herramienta.

Por último, esperamos haber contribuido con las aplicaciones empíricas de las teorías de las finanzas a la realidad de los fondos de inversión de renta variable brasileños. 


\section{Bibliografia}

ASSOCIAÇÃO NACIONAL DOS BANCOS DE INVESTIMENTO (2006). Classificaçäo ANBID. Disponible en www.anbid.com.br, consultado en 30.01.2006.

BOLSA DE VALORES DE SÃO PAULO (2006): Dicionário de Finanças. Disponible en www.bovespa.com.br, consultado en 01.06.2006.

CÂMARA DE CUSTÓDIA E LIQUIDAÇÃO (2006). Taxa DI-CETIP: Metodologia de cálculo acumulado. Disponible en www.cetip.com.br, consultado en 01.06.2006.

COMISSÃO DE VALORES MOBILIÁRIOS (2004). Instrução CVM no 409 de 18 de Agosto de 2004. Dispõe sobre a constituição, a administração, o funcionamento e a divulgação de informações dos fundos de investimento. Disponible en www.cvm.gov.br, consultado en 30.01.2006.

FERRUZ, L y SARTO, J.L (1997a): «Revisión Critica de las Medidas Clásicas de Performance de Carteras y Propuesta de Índices Alternativos: aplicación a Fondos de Inversión Españoles (1990-1995)».Boletín de Estudios Económicos, vol. LII, diciembre, pgs. 550-573.

$$
\text { y __ (1997b): «Análisis Financiero de la Performance de los Fondos de Pensiones en España Durante el Período }
$$
1989-1995».Cuadernos Aragoneses de Economía, vol.7, pgs. 403-415.

y _ (2003): «An Analysis of Spanish Investment Fund Performance: some considerations concerning Sharpe's Ratio». The International Journal of Management Science, noviembre, pgs. 1-12.

JENSEN, M. C. (1968): «The Performance of Mutual Funds in the Period 1945 - 1964».Journal of Finance, vol. 23, mayo, pgs. 383-417.

MARKOWITZ, H (1952): «Portfolio Selection». Journal of finance, Marzo.pgs.77-91

SALVADOR, M. y GARGALLO, P. (2006): Análisis de Datos Multivariantes con SPSS. Análisis de Cluster. Presentación en clase no publicada. Universidad de Zaragoza.

SARTO, J.L. (1995): Valoración de la Gestión de Carteras de los Productos de Inversión Colectiva y de Ahorro-Previsión. La Medida de la Performance. Tesis Doctoral no publicada. Universidad de Zaragoza.

SHARPE, W. (1963): «A Simplified Model for Portfolio Analysis». Science, vol IX, enero, pgs. 277-293.

445-452.

(1964): «Capital Asset Prices: a Theory of Market Equilibrium under Conditions of Risk ».Journal of Finance, septiembre, pgs.

STEVENSON, J. W. (2000): Estatística Aplicada à Administração. Ed. Haper \& Row do Brasil. São Paulo.

TREYNOR, J. L. (1965): «Haw to Rate Management of Investment Funds».Harvard Business Review, enero - febrero, pgs. 63-75.

\section{Dirección de los autores}

Universidad de Zaragoza

Pedro Cerbuna 12

Zaragoza - España

50009 


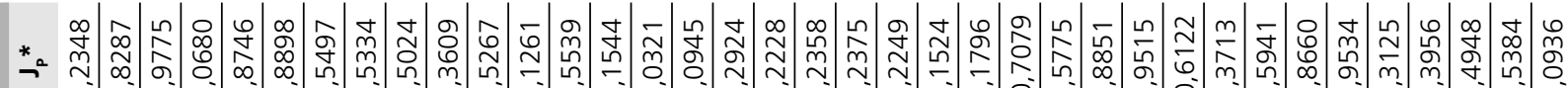

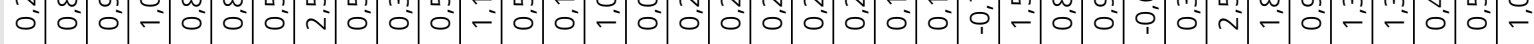

* б

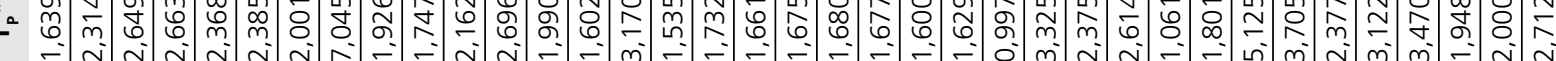

০)

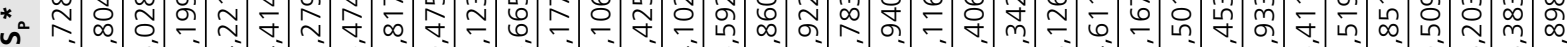

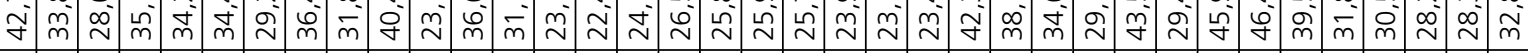

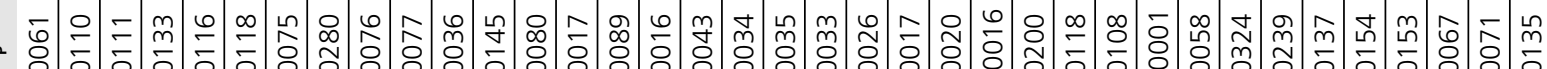

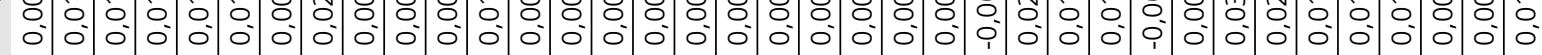

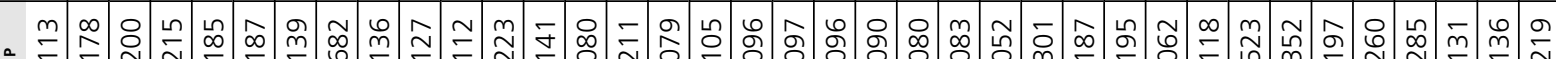

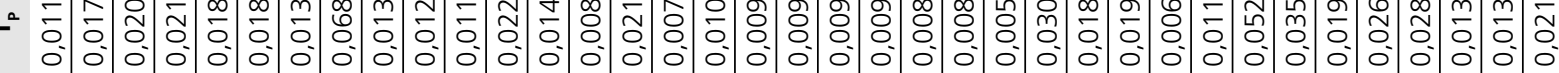

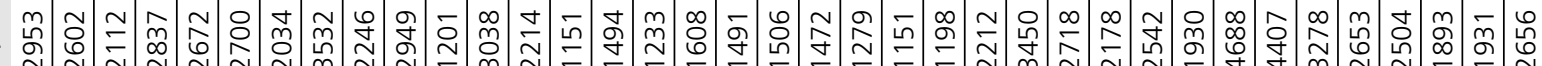

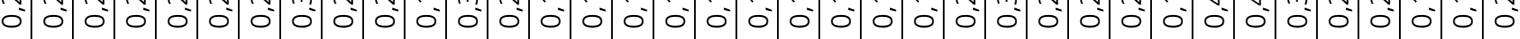

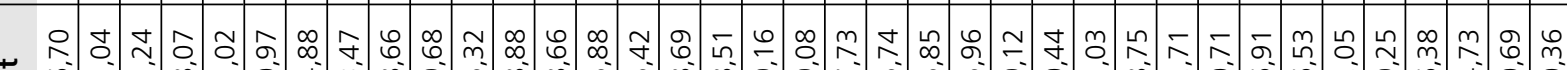

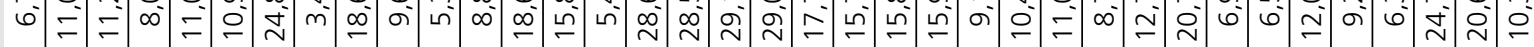

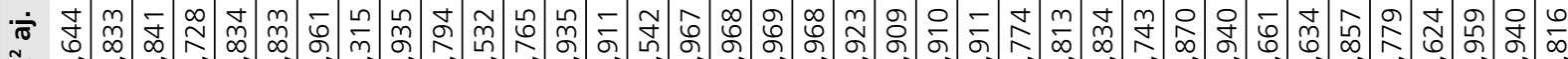

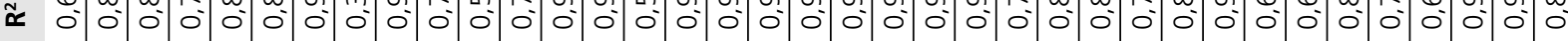
กㅇㅇㅢ

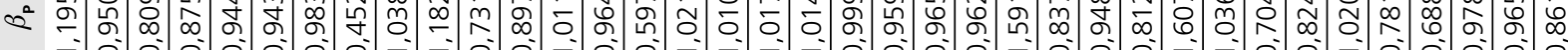

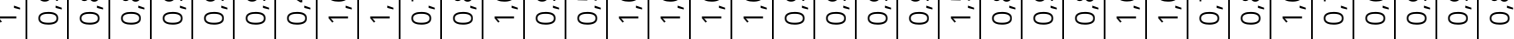

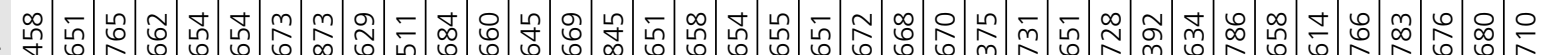

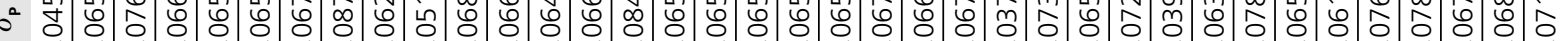

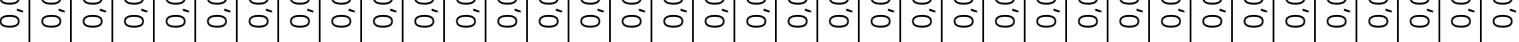

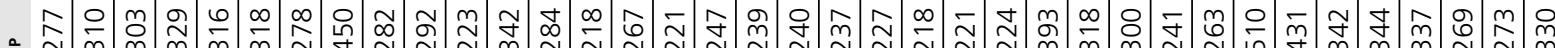

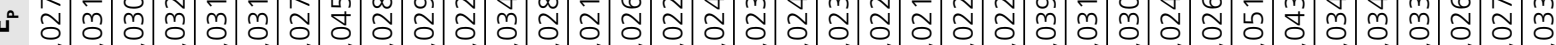

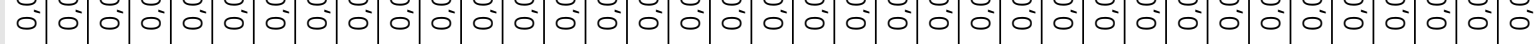

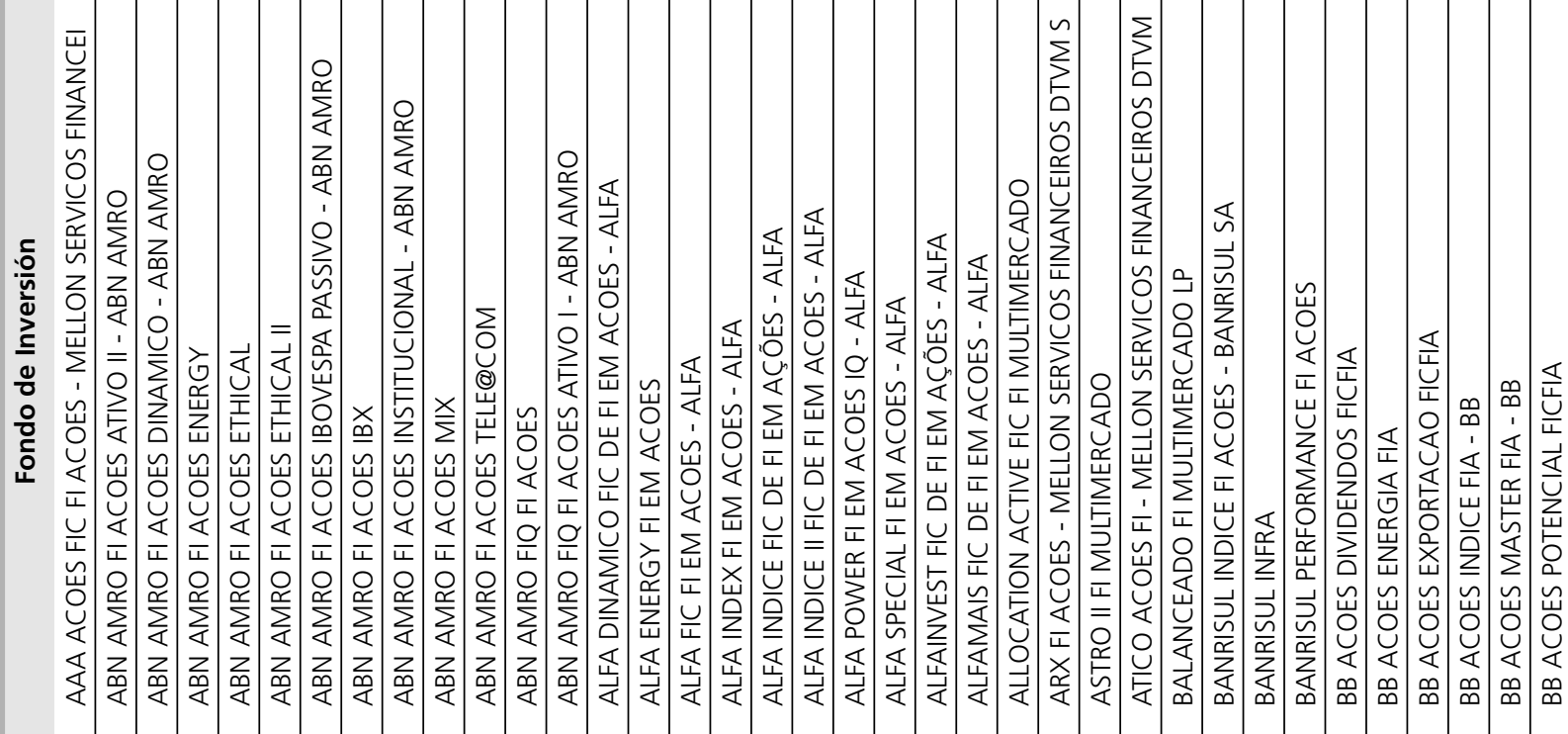




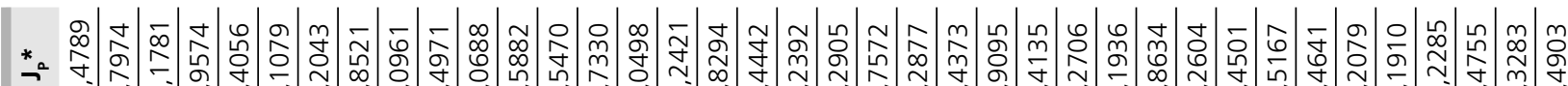

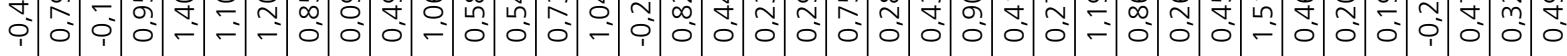

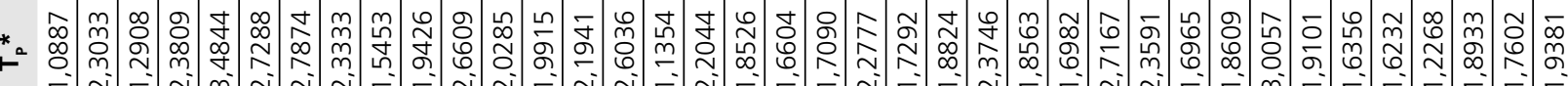
б

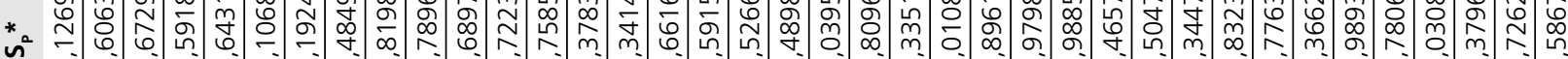

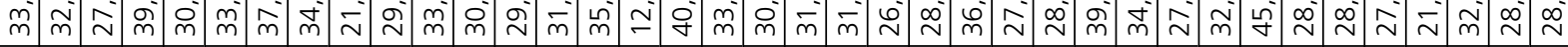

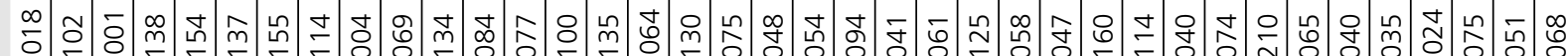
o.

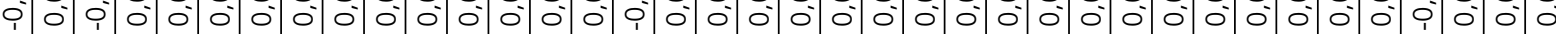

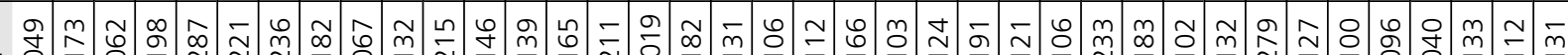

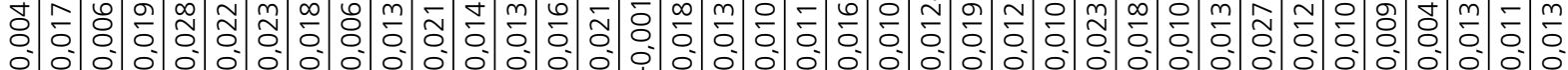

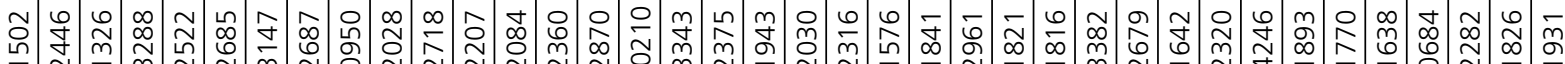

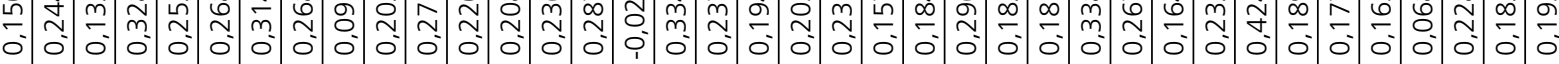
ก

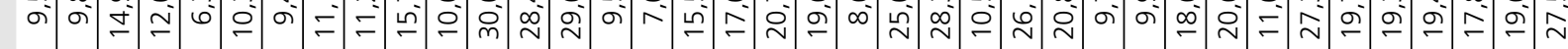

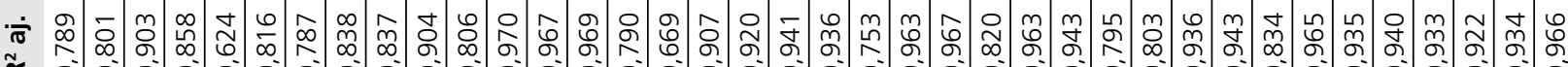

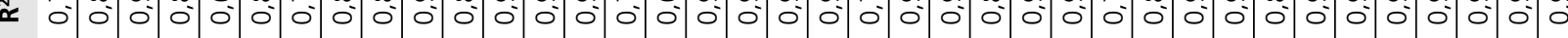
$\tilde{m}$ 直

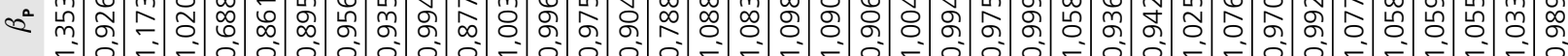

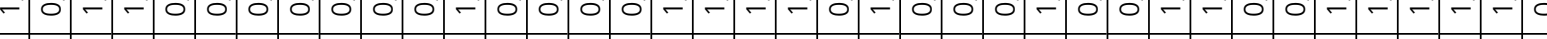

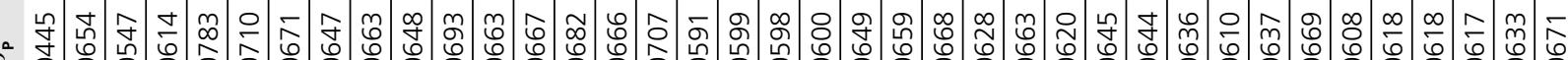

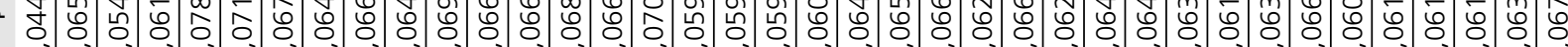

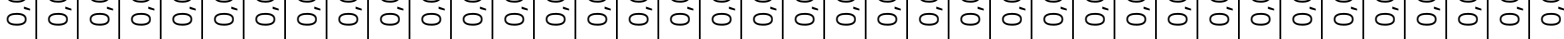
ᄋ - J స̃

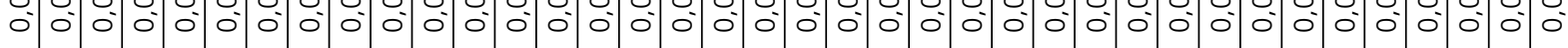
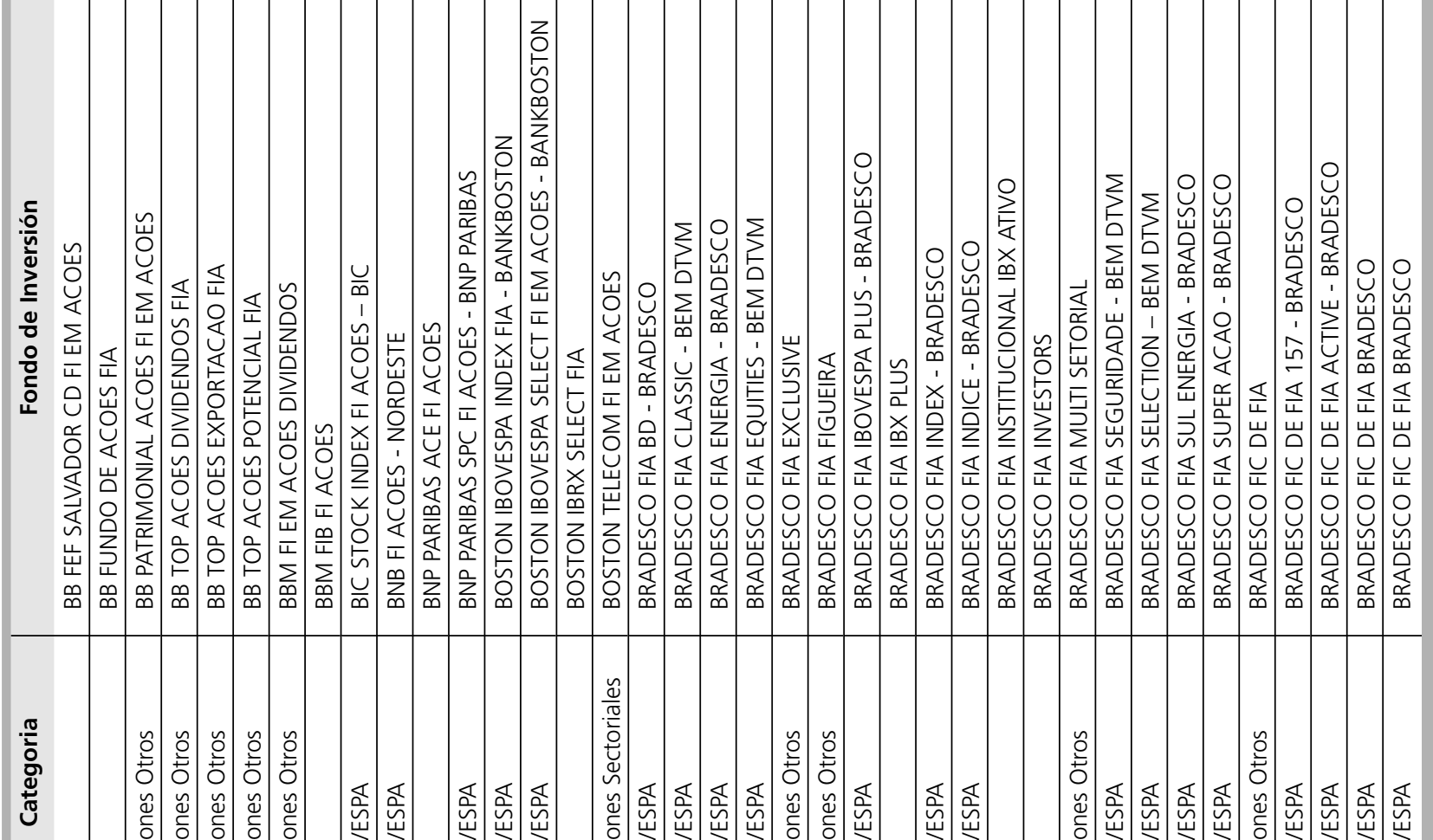

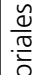
앙

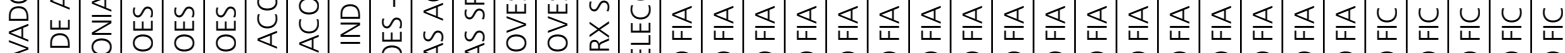

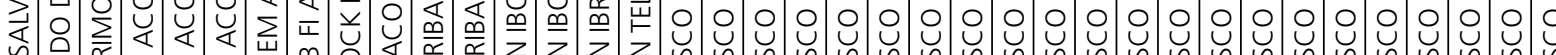

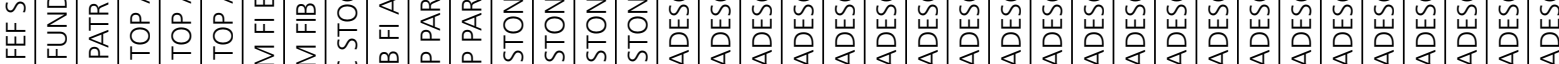

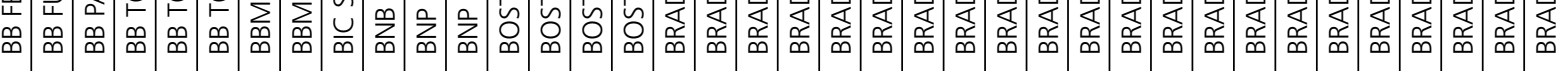

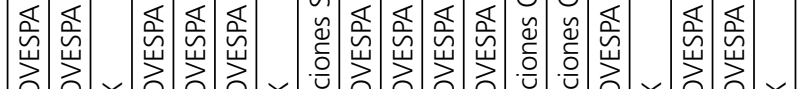

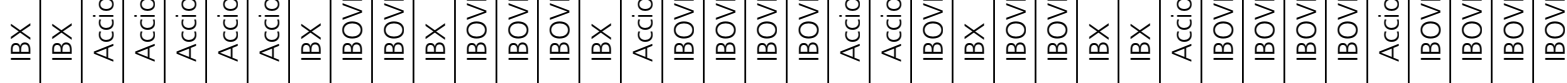




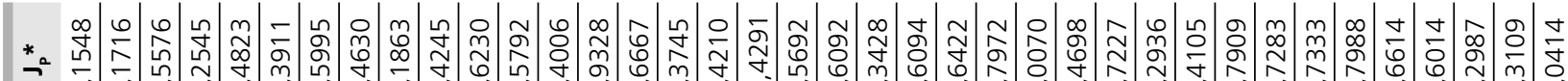

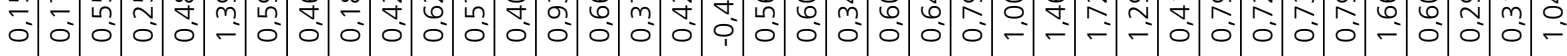

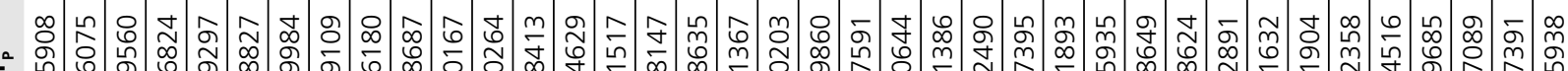

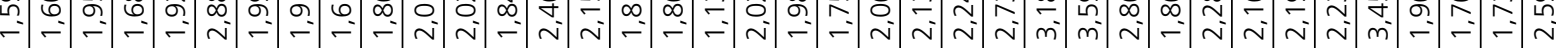

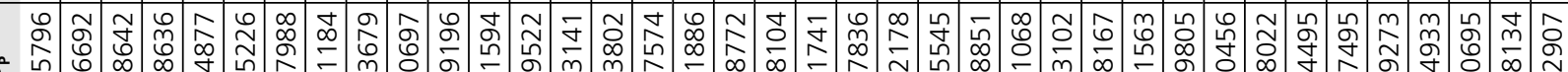

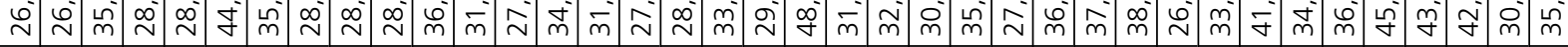

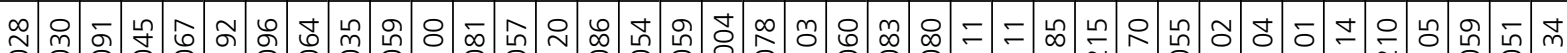

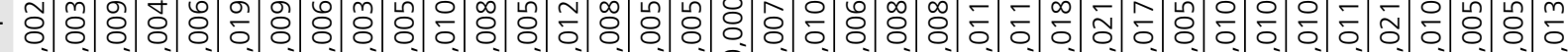

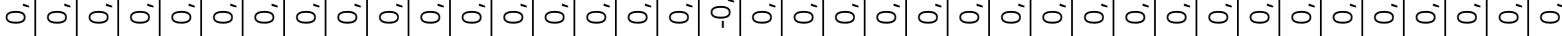

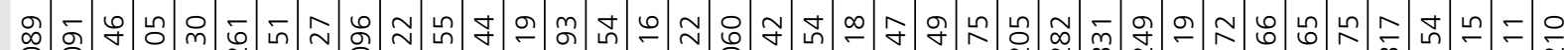

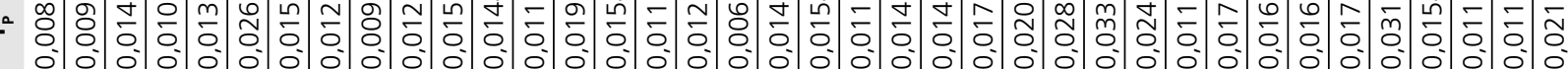

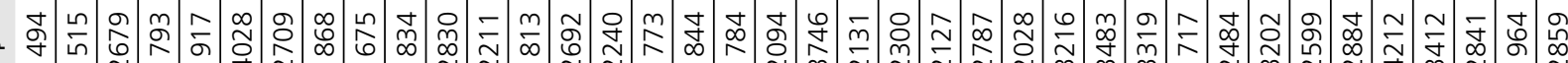

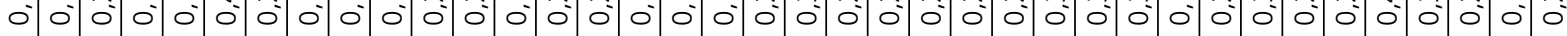

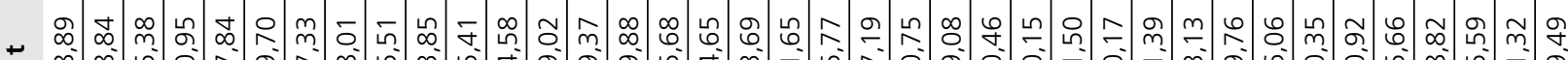

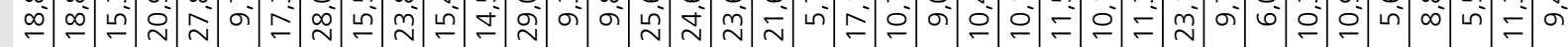

㲾

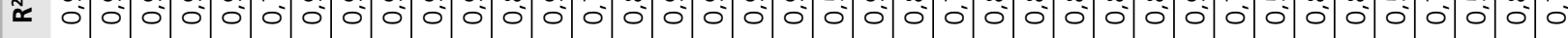
f «

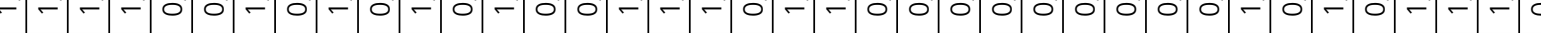

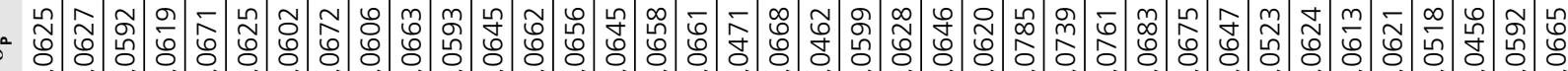

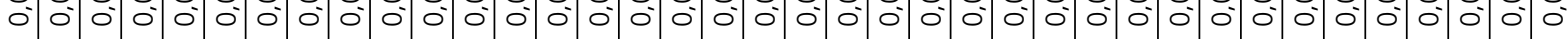

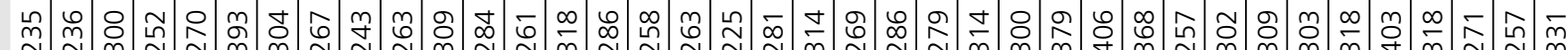

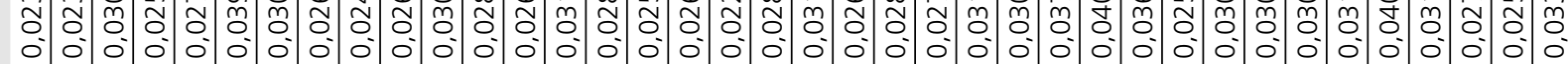

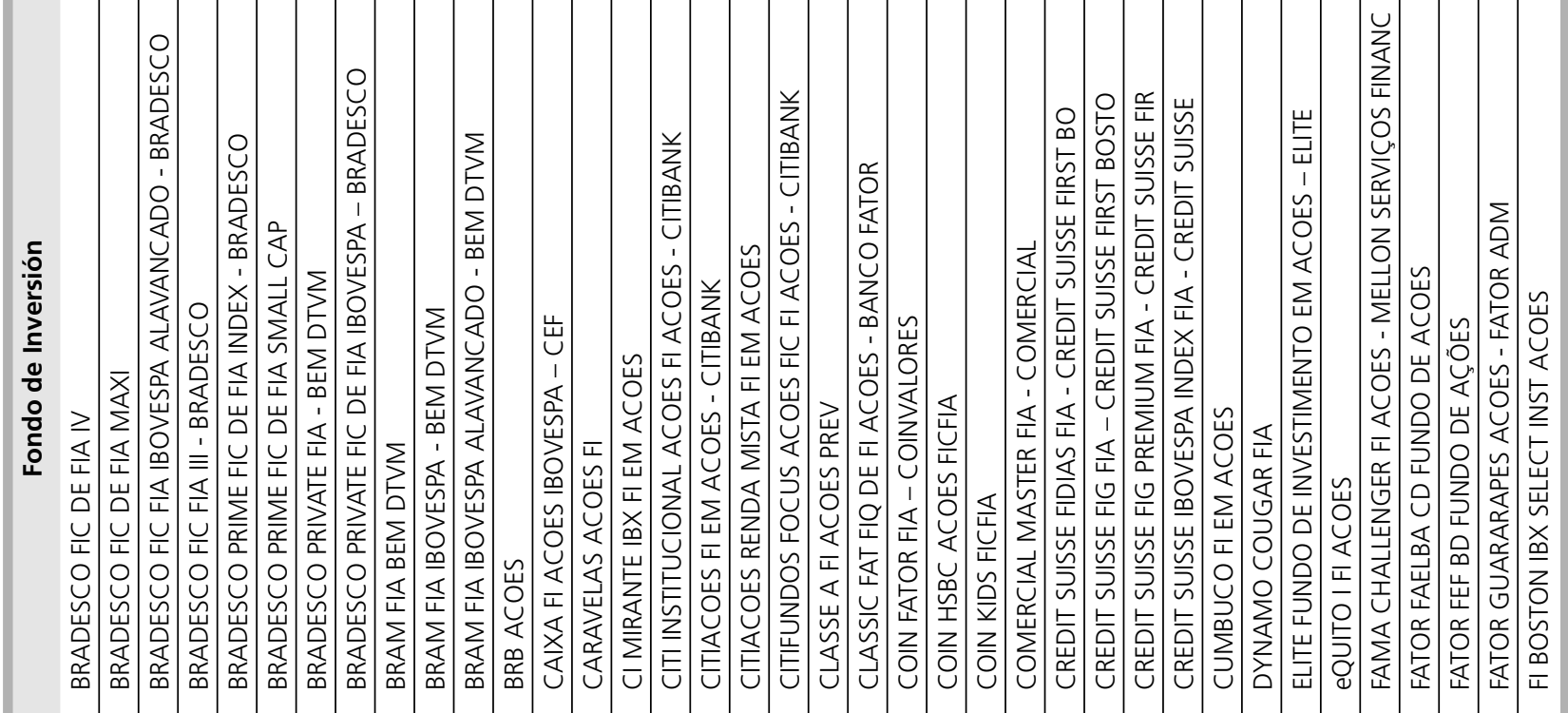

Ith

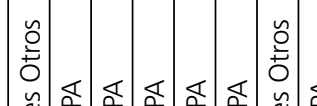

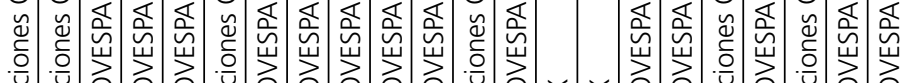

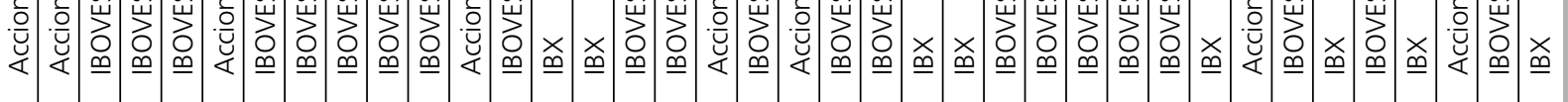




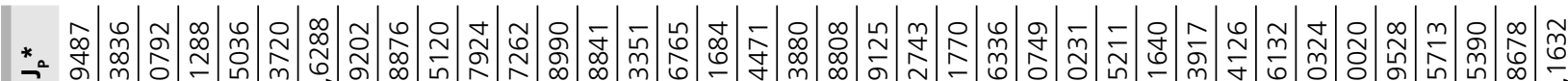

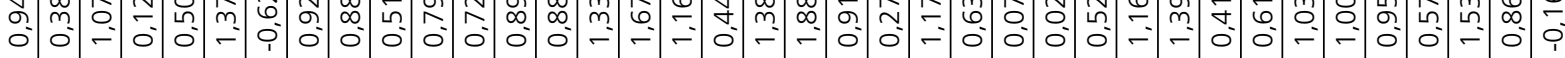

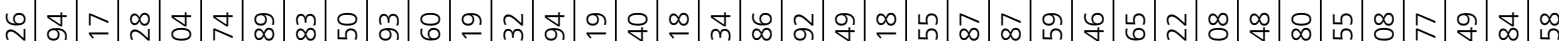

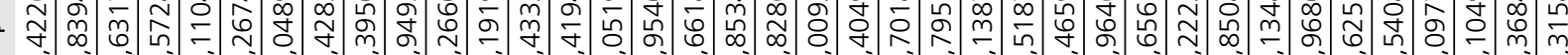

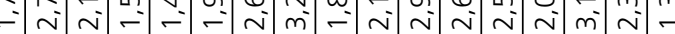
J

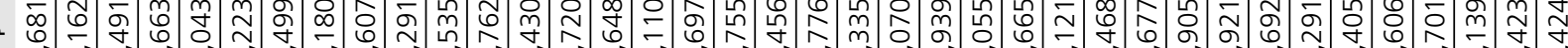

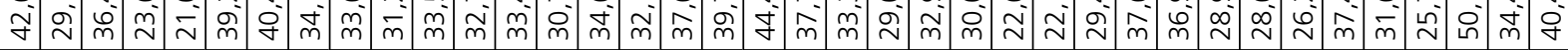
จิ 彳ิ ôे

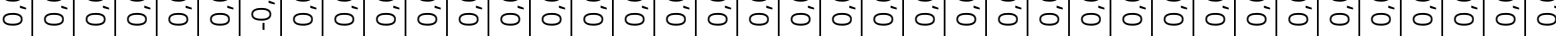

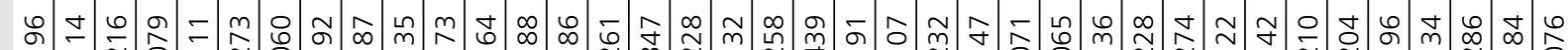

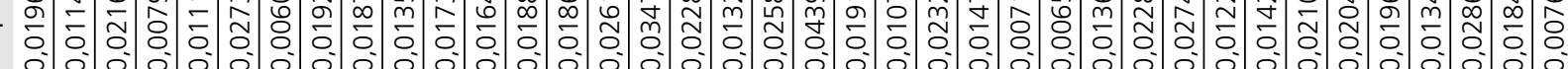

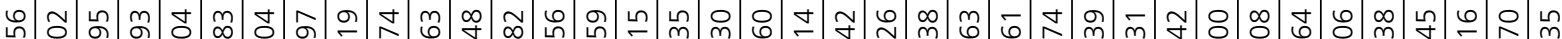
卉

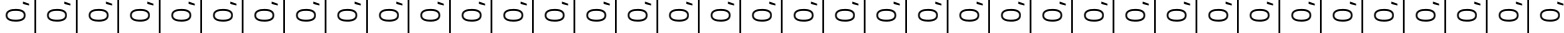

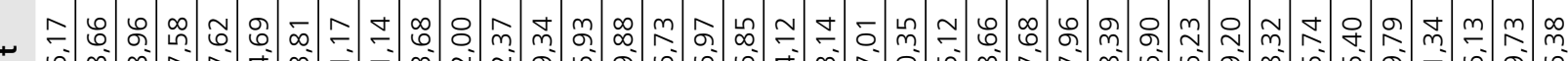
$\therefore$

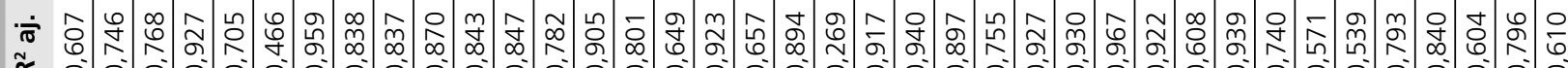

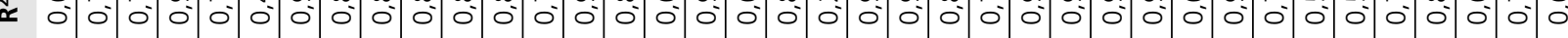
$e^{2}$ o

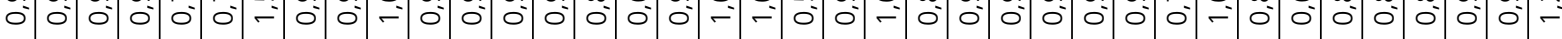

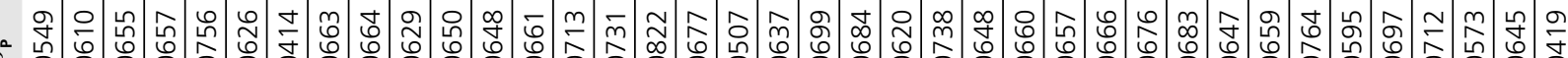
$\circ$.

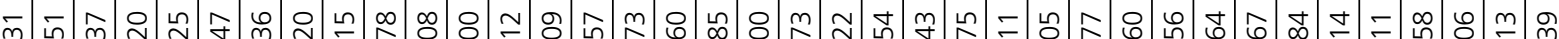

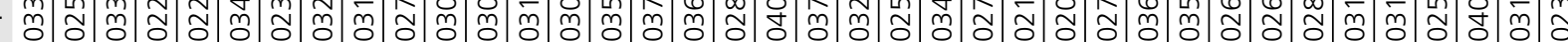

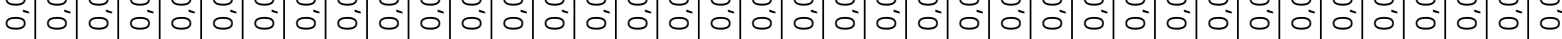

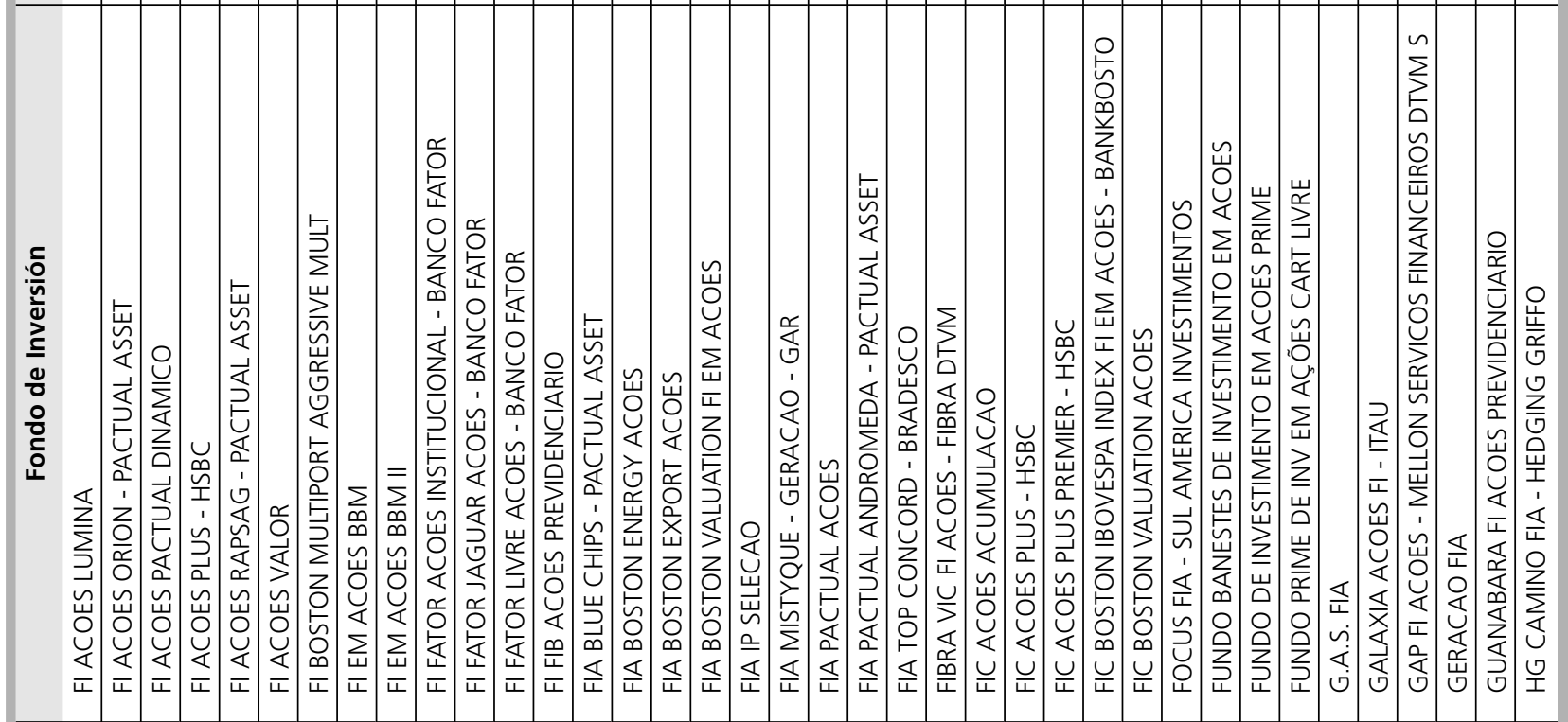

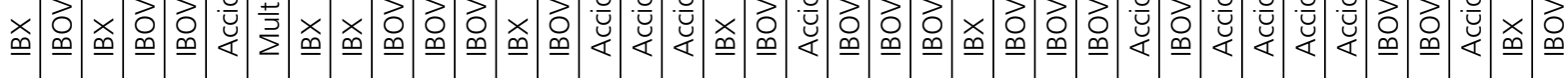




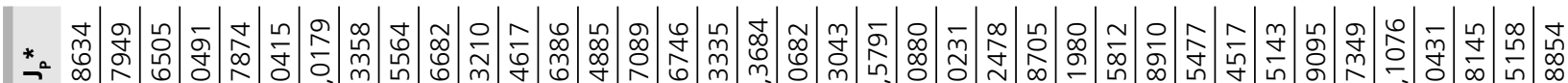

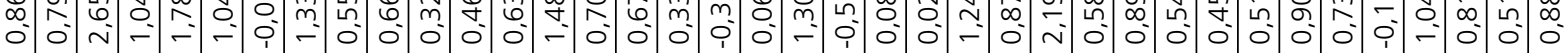

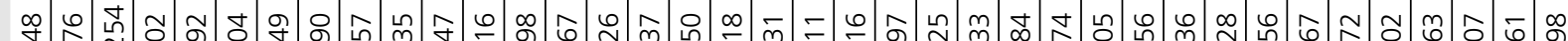

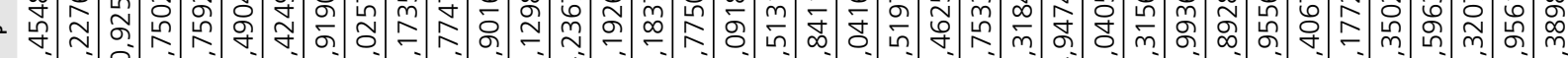

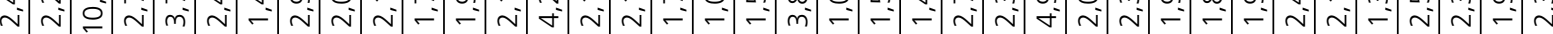
ட

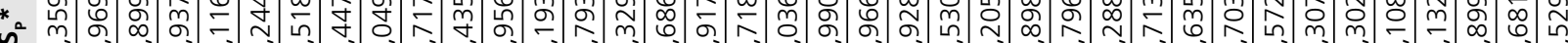

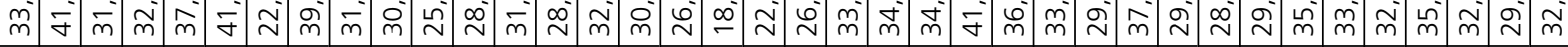
o

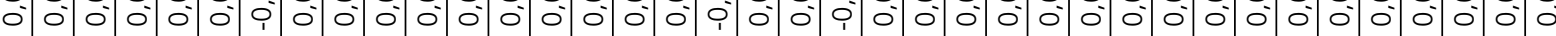

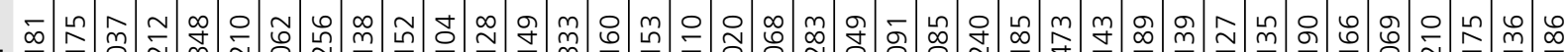

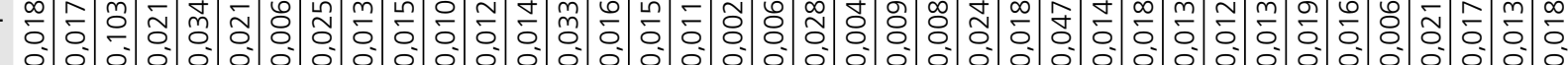

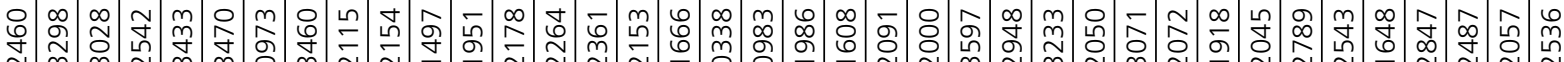

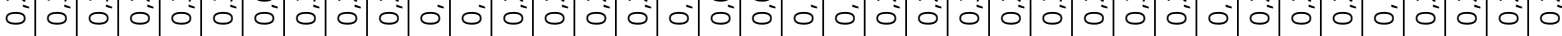

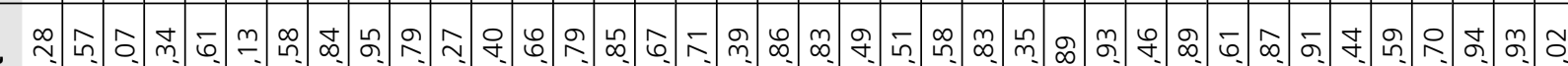

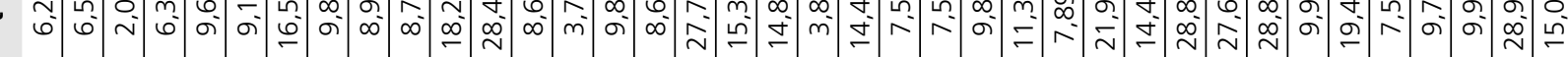

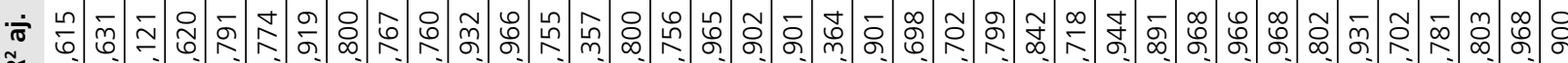

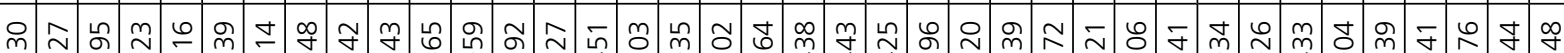

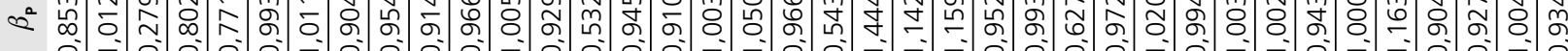

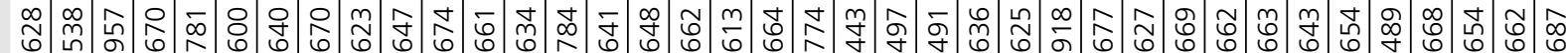
०

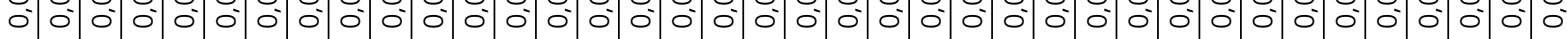

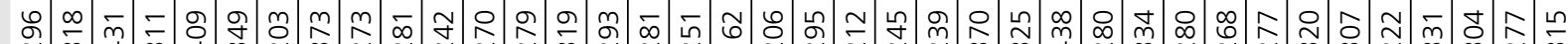

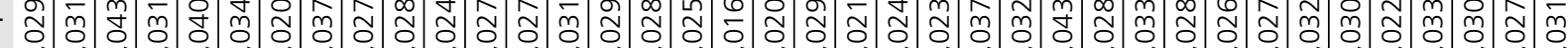

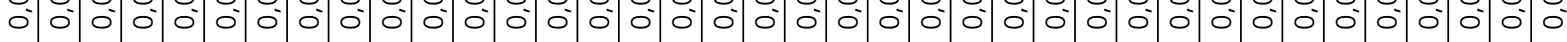

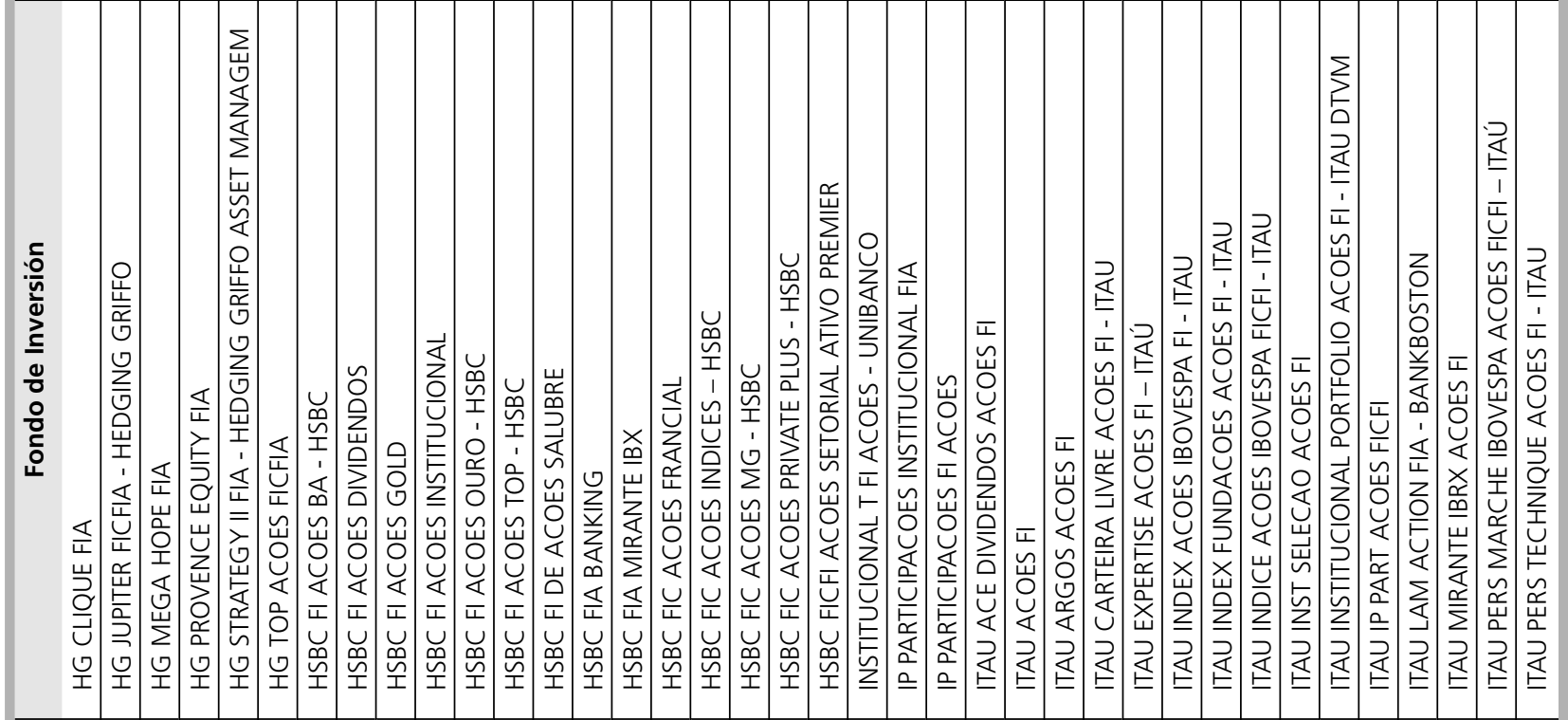

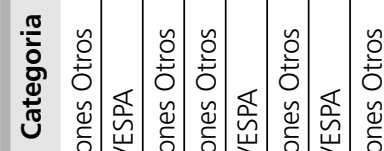

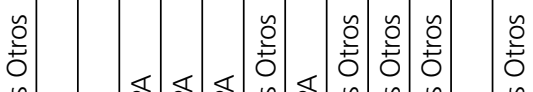

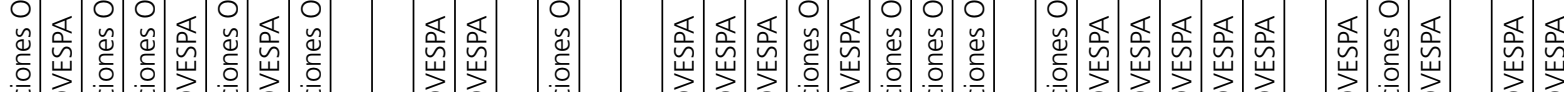
案 


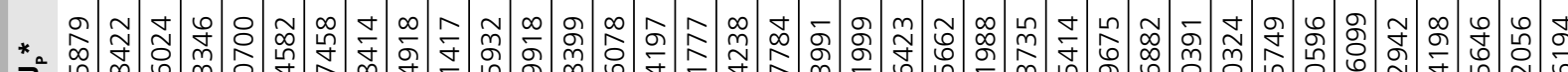

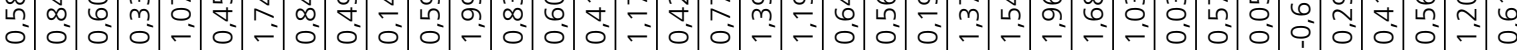

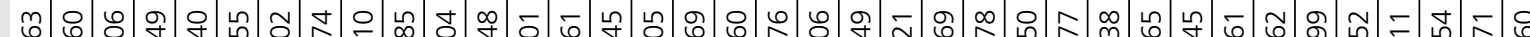
* 子

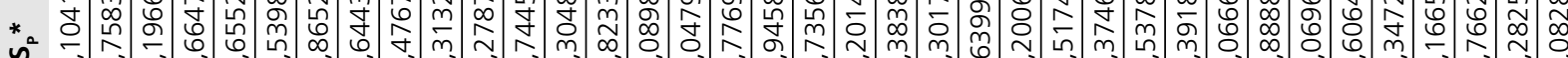

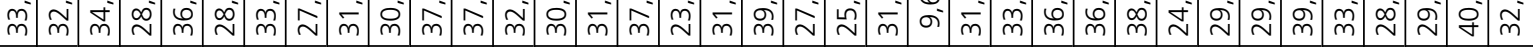

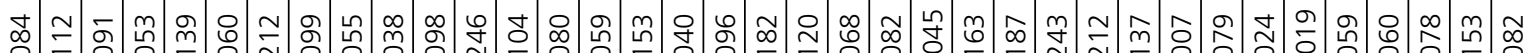

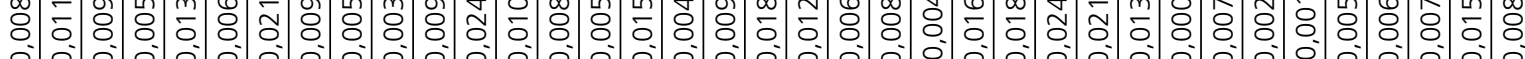

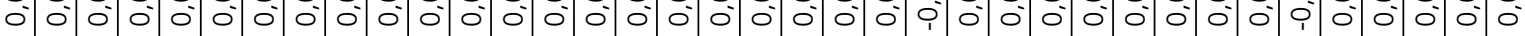
fod

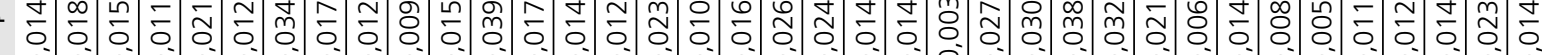

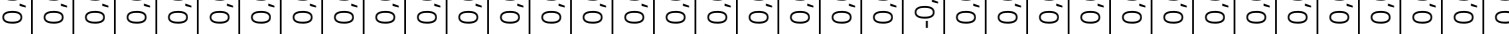

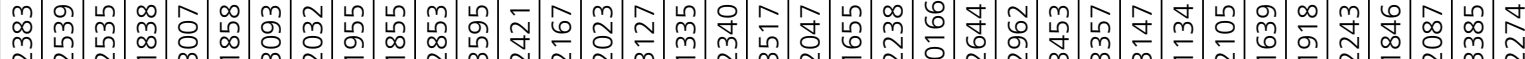

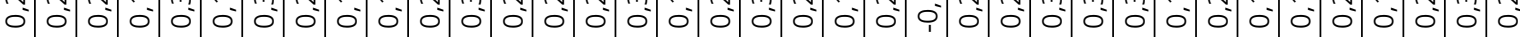

-

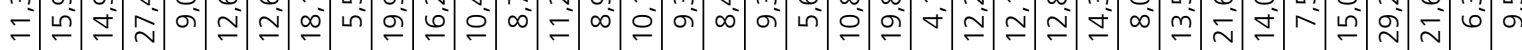

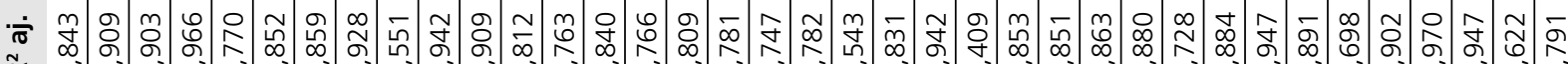

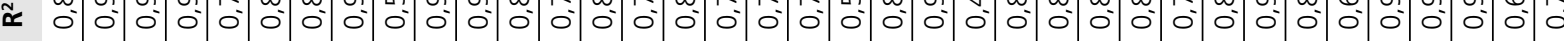

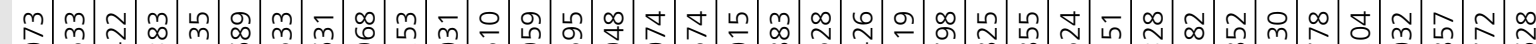

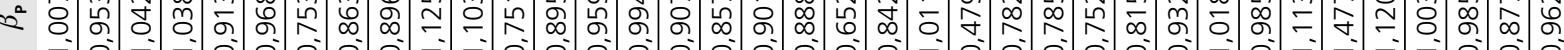

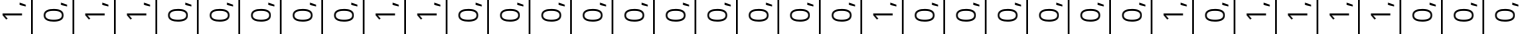

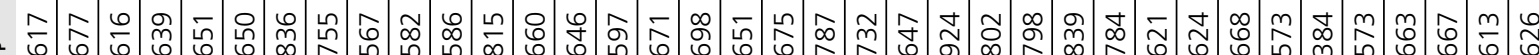

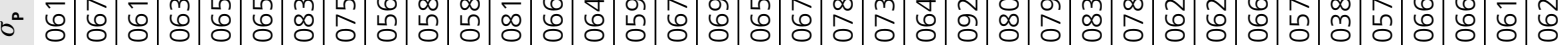

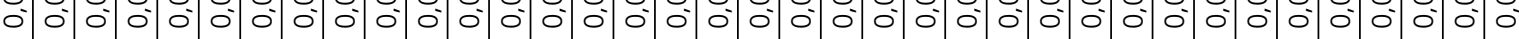

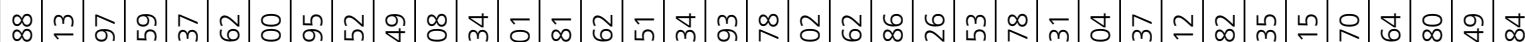

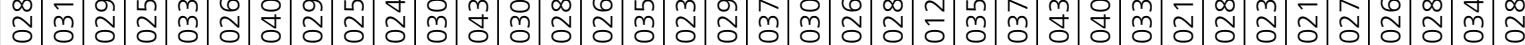

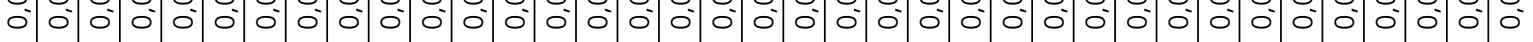

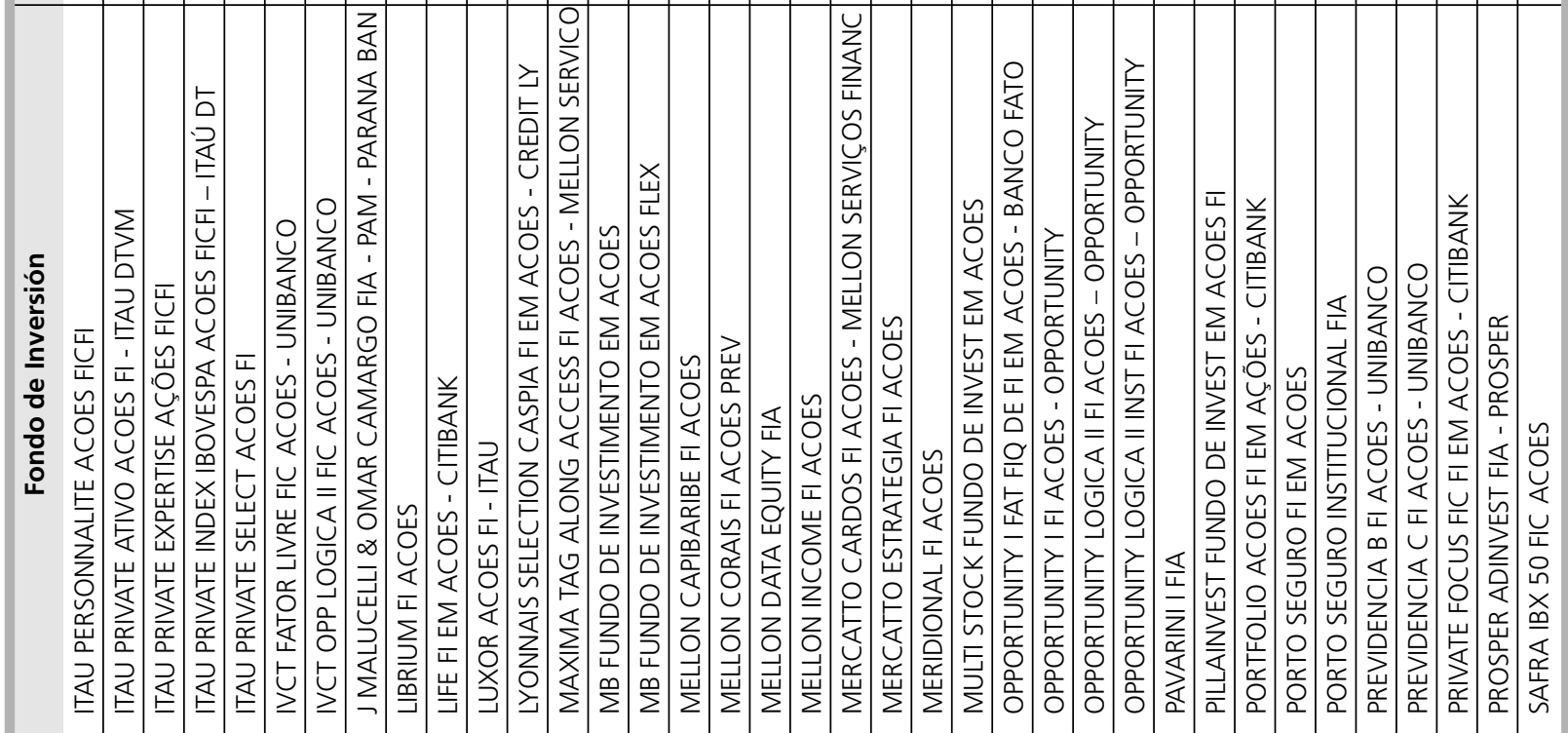

ט.

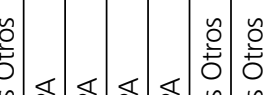

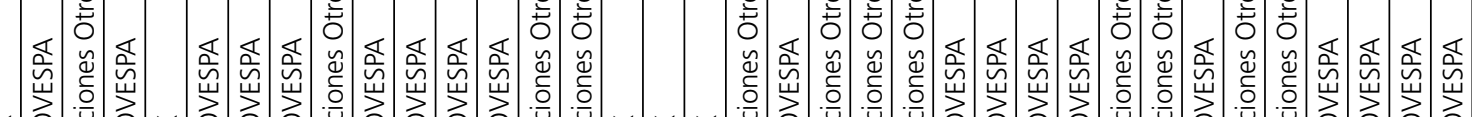

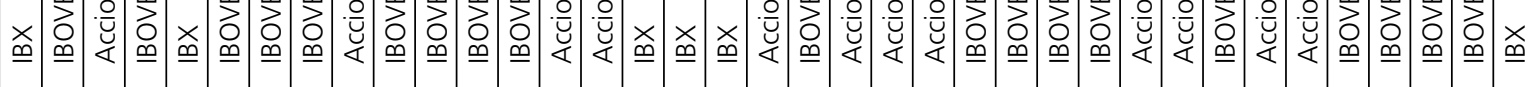




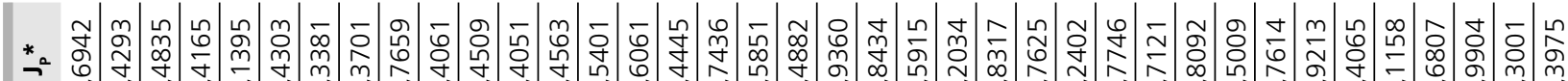

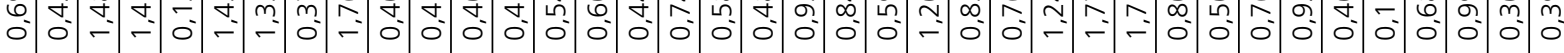

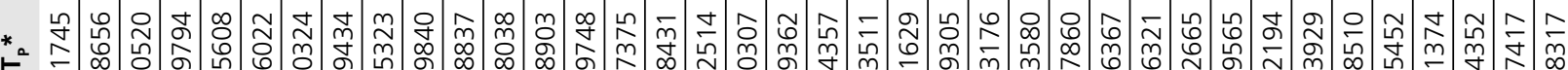

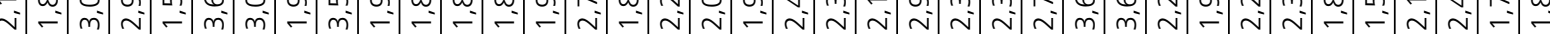

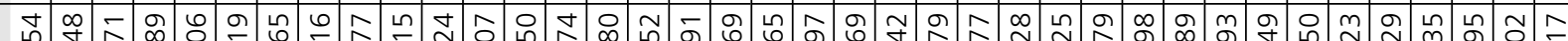

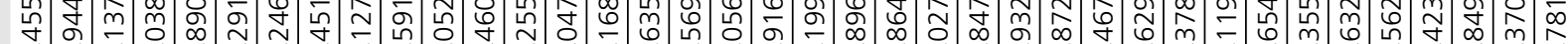

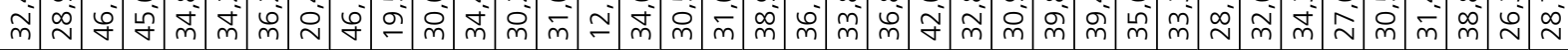
б o

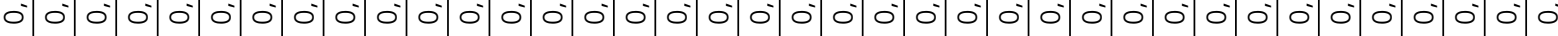

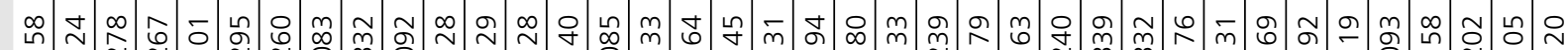

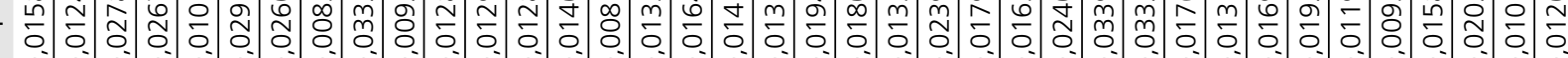

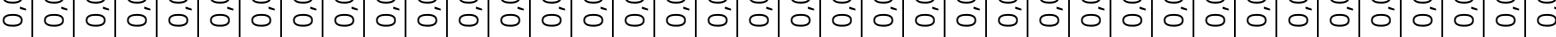

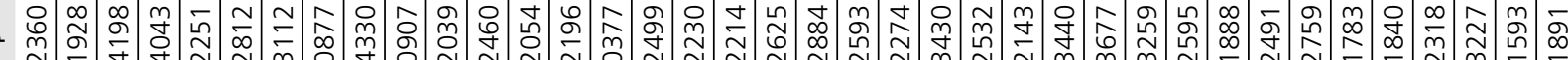

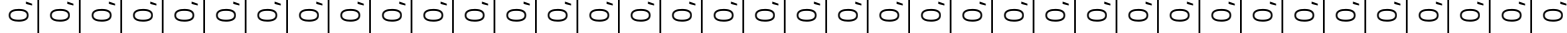

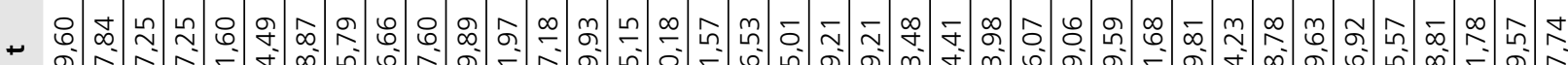

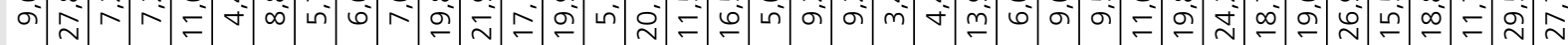

㲾

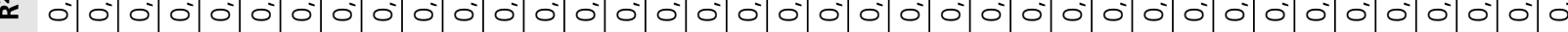

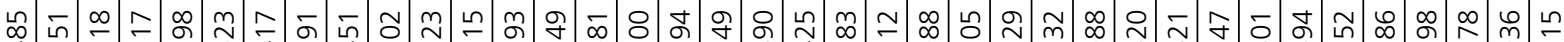

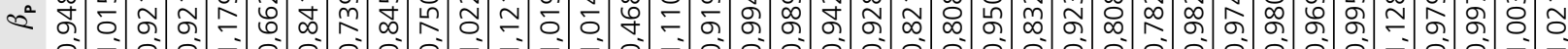

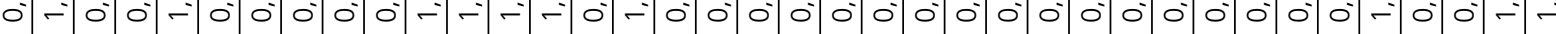

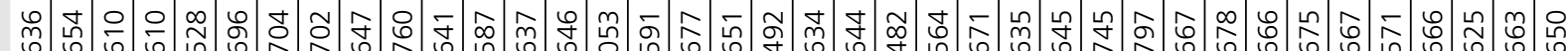
•

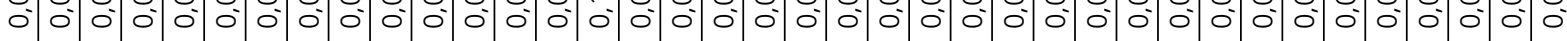

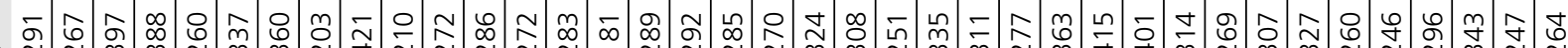
సิ

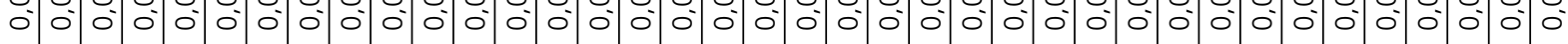

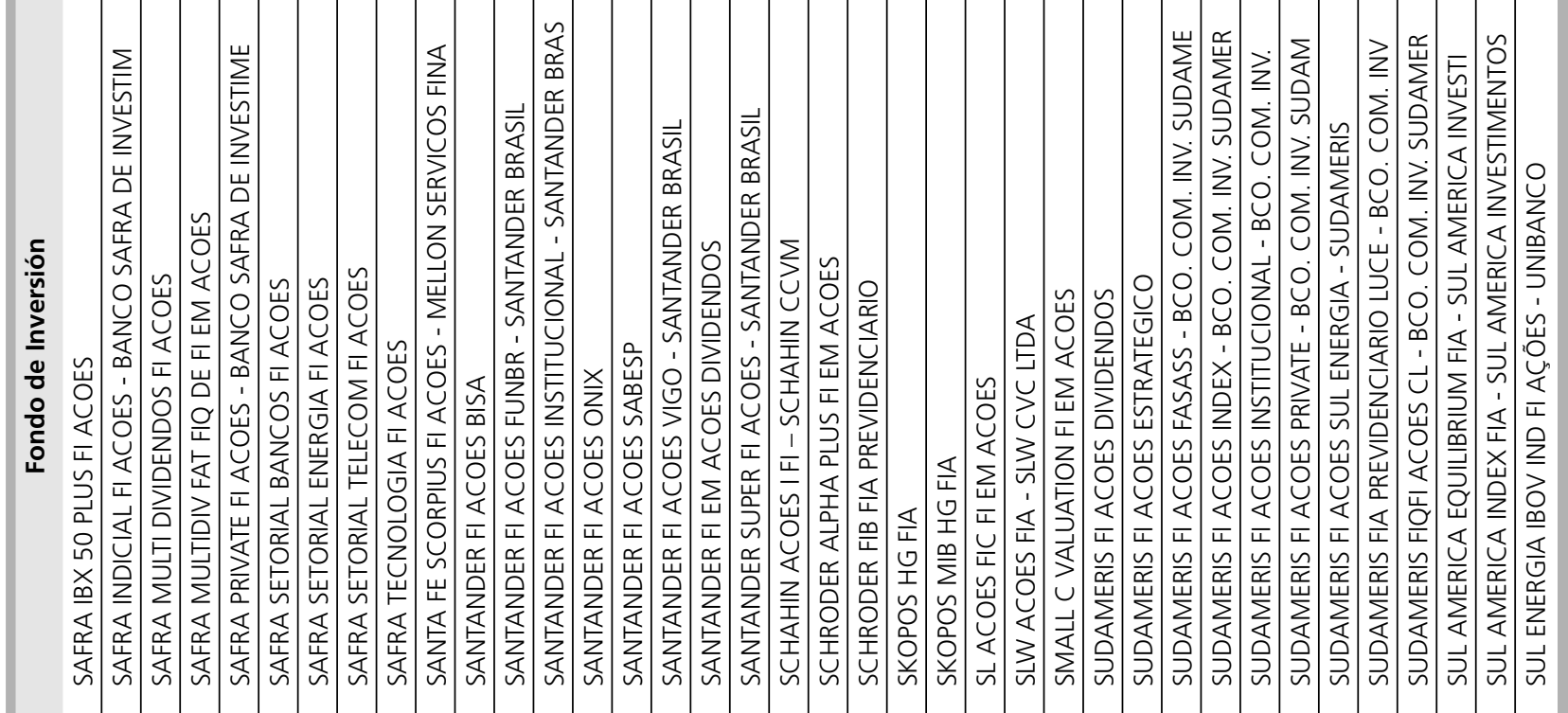

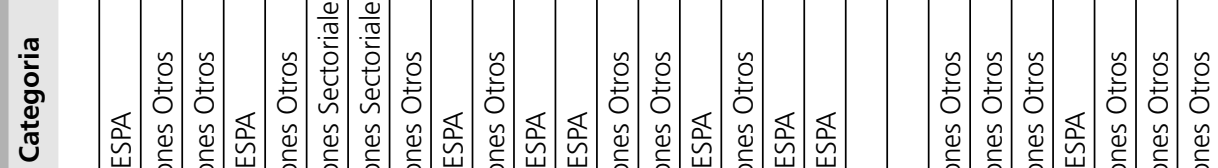

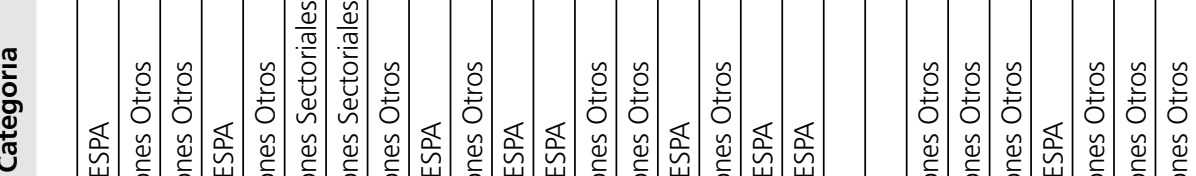

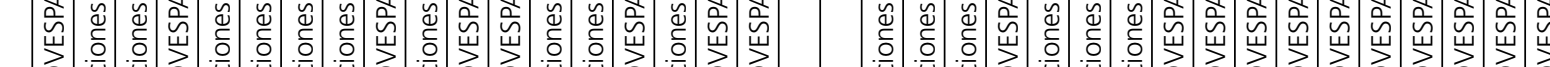

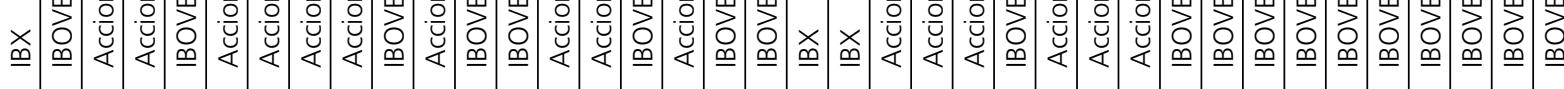


* o

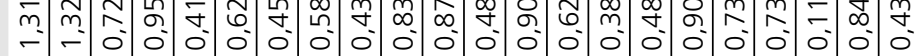

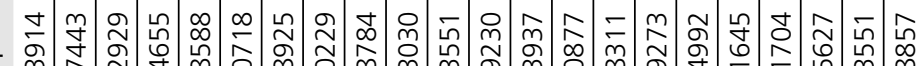

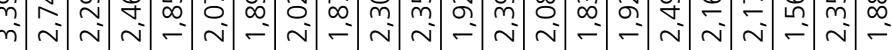

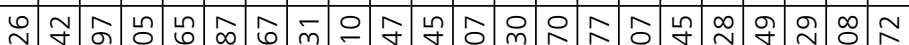

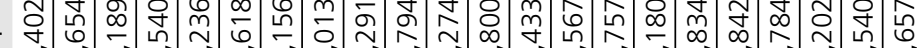
হं テ ○一 $\begin{array}{lllllllllllllllllllllllllll}0 & 0 & 0 & 0 & 0 & 0 & 0 & 0 & 0 & 0 & 0 & 0 & 0 & 0 & 0 & 0 & 0 & 0 & 0 & 0 & 0 & 0\end{array}$ 위유

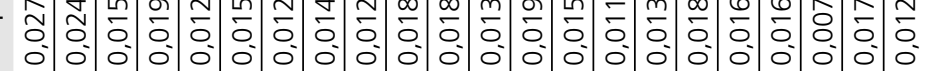
₹

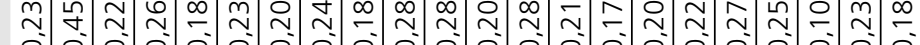

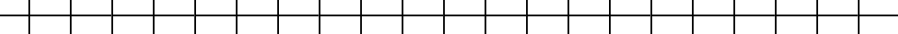

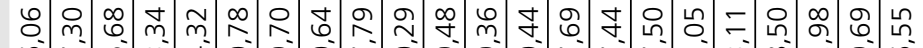

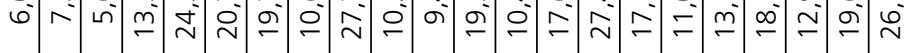

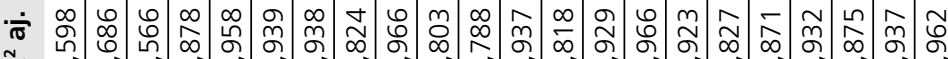

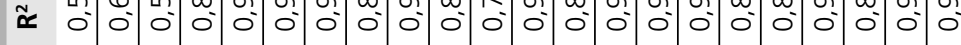

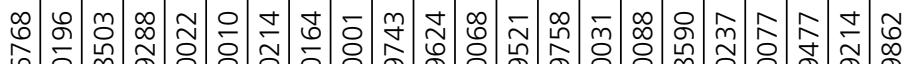

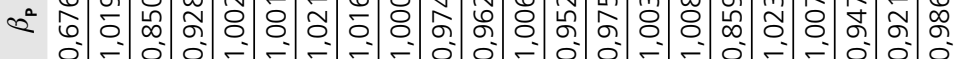
o $\begin{aligned} & 0 \\ & 0\end{aligned}$

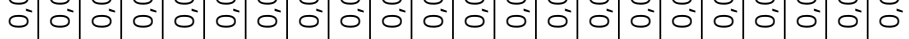
\

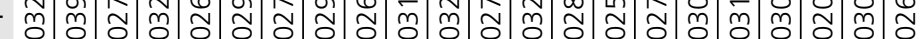

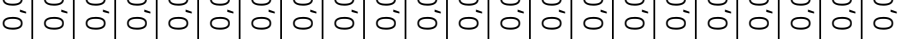
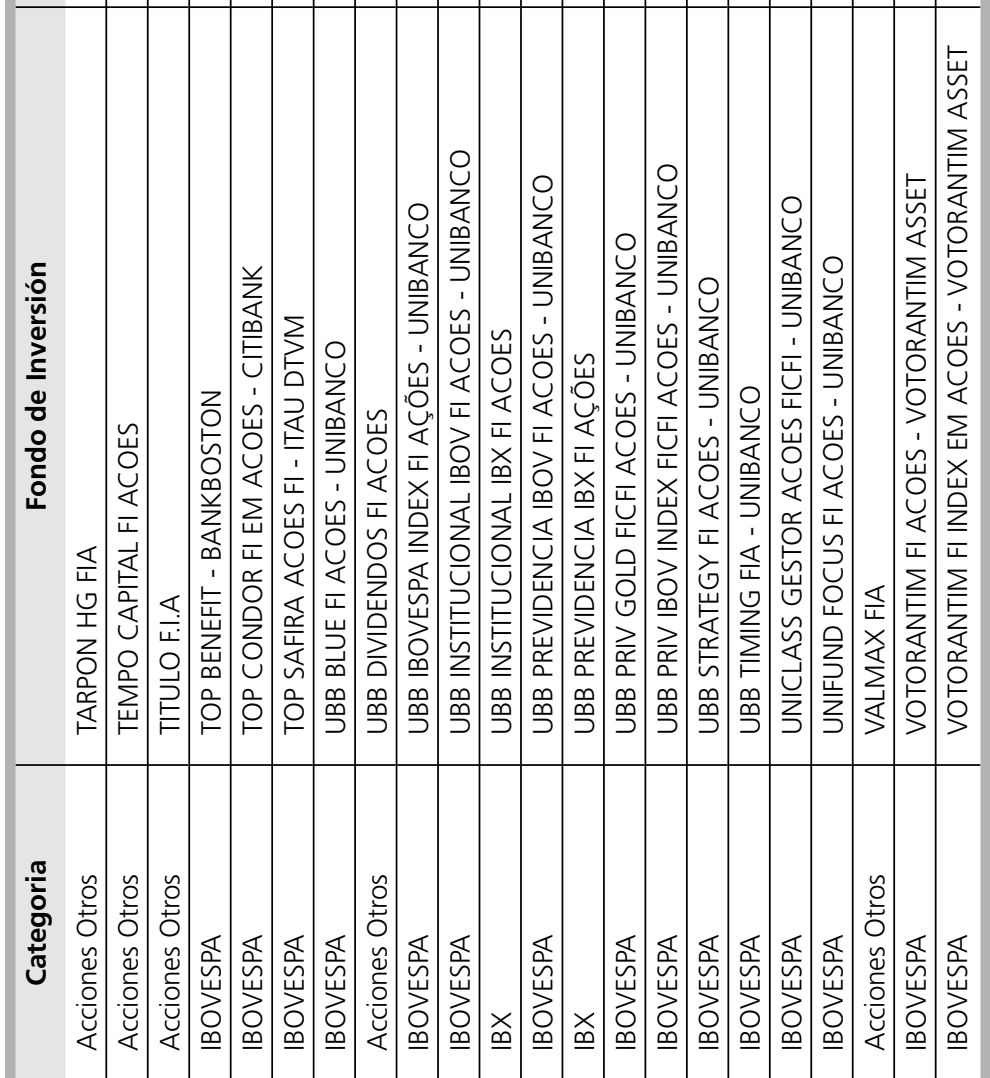